\title{
On the onset of multi-wave patterns in laterally heated floating zones for slightly supercritical conditions
}

\author{
Marcello Lappa ${ }^{1}$ \\ ${ }^{1}$ Department of Mechanical and Aerospace Engineering, University of Strathclyde, James Weir \\ Building, 75 Montrose Street, Glasgow, G1 1XJ, UK - email: marcello.lappa@strath.ac.uk, \\ marlappa@unina.it
}

Abstract: This analysis follows and integrates the line of inquiry started in past author's works (Phys. Fluids, 15(3): 776-789, 2003, and Phys. Fluids 16(2): 331-343, 2004) about the typical instabilities of Marangoni flow and associated hierarchy of bifurcations in laterally heated floating zones with various shapes and aspect ratios. The main motivation for re-examining this kind of problems, which so much attention have attracted over the last twenty years, is the recent discovery (Kudo, Ueno and Kawamura, (2014), in Japanese, DOI: 10.1299/transjsme.2014tep0095) of a chaotic state in region of the space of parameters where on the basis of existing theories and earlier results for the classical liquid-bridge problem with organic fluids, the flow should be relatively regular in time and with a simple structure in space. Axisymmetric computations are used to obtain the steady basic state, and then the Navier Stokes equations are solved in their complete, threedimensional, time-dependent and non-linear formulation to investigate the evolution of azimuthal disturbances. It is shown that the "apparent" doubling or quadrupling of the azimuthal wavenumber in the equatorial plane, previously reported for the case of floating zones of liquid metals, is replaced for high-Prandtl-number liquids by the complex interaction of disturbances with distinct spatial and temporal scales. These disturbances become critical at relatively comparable values of the Marangoni number. The unexpected multiplicity of waveforms and competition of spatial modes is explained according to the increased complexity of the considered system in terms of flow topology and structure with respect to the classical half-zone configuration.

Key words: Marangoni flow, Lateral heating, Instability and bifurcation in Fluid Dynamics, Chaos

\section{Introduction}

Marangoni flows and related instabilities have attracted much attention over recent years ${ }^{1-6}$. Zone melting (or zone refining or floating zone process, FZ) is a group of similar methods, specifically conceived for the purification of crystals, in which such flows play a fundamental role. With such methods, a short portion of a rod of an initially impure or polycrystalline material is melted, and the molten zone is moved along the rod. The liquid region grows at expenses of impure solid at its forward edge and leaves a wake of purer solid behind it as it moves along the bar. As the liquid cools, the material crystallizes on the seed-part of the rod.

Since the melt never comes into contact with anything but vacuum (or inert gases), there are no impurities that the melt may incorporate by dissolving the crucible material as it would be in the Czochralski (CZ) crystal growth method. Accordingly, materials with higher purity and less contamination can be obtained (see, e.g., Benz ${ }^{7}$ ). 
With a growing number of facilities around the world this technique is gaining popularity as the method of choice for the growth of a wide range of materials, including metals, semiconductors (Cröll et al., ${ }^{8}$ ), high-temperature superconductors, new magnetic materials and a variety of conventional and nonconventional oxides (Saurat and Revcolevschi ${ }^{9}$, Shindo et al., ${ }^{10}$; Shindo ${ }^{11}$; Balbashov and Egorov ${ }^{12}$; Kimura and Kitamura ${ }^{13}$; Revcolevschi and Jegoudez ${ }^{14}$; Moest et al., ${ }^{15}$ ). As an example, the list of oxide materials includes simple oxides such as $\beta-\mathrm{Ga}_{2} \mathrm{O}_{3}$ or $\mathrm{TiO}_{2}$, as well as, complex oxides such as the spin Peierls material $\mathrm{CuGeO}_{3}\left(\right.$ Revcolevschi and Jegoudez $\left.{ }^{14}\right)$. Although, high-quality crystals of $\beta-\mathrm{Ga}_{2} \mathrm{O}_{3}$ as large as 2.5 centimeters in diameter have been reported by Villora et al., ${ }^{16}$, oxides grown by this technique for industrial applications are essentially $\mathrm{Y}_{3} \mathrm{Fe}_{5} \mathrm{O}_{12}$ (Balbashov et al., ${ }^{17}$; Shindo et al., ${ }^{10}$; Balbashov and Egorov ${ }^{12}$ ) and the abovementioned $\mathrm{TiO}_{2}$ (Higuchi and Kodaira ${ }^{18}$ ).

Detailed historical notes about the development of FZ technique can be found in Dabkowska and Dabkowski $^{19}$ and an earlier paper by Dabkowska and Gaulin ${ }^{20}$. Here we limit ourselves just to observing that the main difference between metallic materials and oxides lies essentially in the related value of the Prandtl number (Pr defined as the ratio of the melt kinematic viscosity to its thermal diffusivity, which is generally $<1$ for the former materials and $>1$ for the latter). Another difference comes from the macroscopic appearance of these materials (liquid metals and semiconductor melts are generally opaque while oxide melts tend to be transparent to visible light). Another important distinction must be introduced with regard to the typical flow instabilities that can emerge in the melt during the processing of the material. Such instabilities have detrimental effects which tend to lower the quality of crystals. It is known that their nature, the hierarchy of bifurcations and ensuing effects depend on the kind of material considered, namely the aforementioned Prandtl number.

The amount of existing literature on such subjects is really impressive (especially if one considers what has been done for the so-called half-zone liquid bridge, a popular simplified model of the FZ process, Refs [1-6] and references therein). Here, due to page limits we limit ourselves to recalling the points still requiring attention, and recent (still unexplained) findings. Along these lines, we start from the simple realization that while there has been an intensive effort to model the FZ processing for the case of metals and semiconductors in the full-zone configuration (Baumgartl et al., ${ }^{24}$; Croll et al., ${ }^{8}$; Lappa ${ }^{22-25}$ Minakuchi et al., ${ }^{26}$; Houchens and Walker ${ }^{27}$; Gelfgat et al., ${ }^{28}$; Lin et al., ${ }^{29}$ ), only a few modeling attempts have been appearing in the literature to understand what is happening inside oxide molten zones (Chen, and $\mathrm{Chieh}^{30}$; $\mathrm{Lan}^{31}$ ).

More recently, Bouizi et al., ${ }^{32}$ investigated the stability of the axisymmetric basic state, with respect to 3D perturbations over a large range of Prandtl number values including both categories of materials $\left(10^{-3} \leq \operatorname{Pr} \leq 10^{2}\right)$. This important analysis confirmed that the main findings related to more than 30-years of studies on the companion liquid-bridge problem can be qualitatively applied to the effective FZ processing technique, i.e. the first flow bifurcation is of a stationary type for $\operatorname{Pr}<<1$, whereas for large Prandtl number values $(\operatorname{Pr}>0.3)$ the first bifurcation implies the onset of oscillatory flow. 
Most recently, however, some authors (Kudo et al., ${ }^{33}$ ) reported on the existence of a chaotic state in a parameter regime where on the basis of existing theories and earlier results for the liquid bridge, the flow should be relatively regular in time and with a simple structure in space (single-frequency and single wavenumber flow).

Following an approach similar to that undertaken by Sakurai et al., ${ }^{34}$, these authors carried out a series of experiments using a substance (silicone oil with $\operatorname{Pr}=28.1$ ) that is liquid at ambient temperatures. A fluid zone was formed between two horizontal supporting disks (made of metal or of a transparent material) with heat being provided to the liquid through its free surface by means of a ring heater. The simplicity of the configuration and the great advantage coming from the utilization of a material that is transparent and in a liquid state at ordinary temperature allowed these researchers to visualize convection by tracer particles from different perspectives. In this way, Kudo et al., ${ }^{33}$ succeeded in identifying precisely the flow transition point and the modal structures in the oscillatory flow. Most surprisingly, although slightly supercritical conditions had been considered, the dominant modal structures was found to be a peculiar combination of disturbances with different azimuthal wavenumbers $m=1,2$, and 3 (a superposition of distinct waves with different spatial modes). In a rather unexpected way, these modal structures were observed to have the characteristics of a standing or a travelling wave, changing from one to another irregularly with the resulting dominant modal structures not depending on the aspect ratio (another important distinguishing feature with respect to earlier studies dealing with the classical liquid-bridge problem $^{1-6,35}$ ).

Motivated by these unexpected results as well as by the general lack of numerical results for floating zones of high-Pr materials (full-zone configuration) here we concentrate on the mathematical modeling and analysis of such experiments. In particular, the problem is investigated by means of numerical solution of the time-dependent Navier-Stokes equations in their complete formulation (three-dimensional, non-linear and time-dependent).

\section{Physical and mathematical model}

\section{A. Modeling the floating zone: "Full zone" configuration}

Two model geometries can be used to simulate the flow in a floating zone: "half zone" and "full zone". A complete and exhaustive description of these models can be found in Ref. 24. Here we simply recall that the half zone (a portion of liquid held between two disks at different temperatures and with an adiabatic liquid/gas interface) is supposed to model the flow in a half of the real floating zone under the assumption that heat is provided to the fluid essentially along the axial direction and under the constraint that the flow is symmetric by reflection about the midplane. With the full zone (a column of liquid held between two disks at the same temperature and heated through the free surface) such restrictions are removed (which makes this geometrical model more effective in reproducing some of the typical features of the real processes used for crystal growth). 


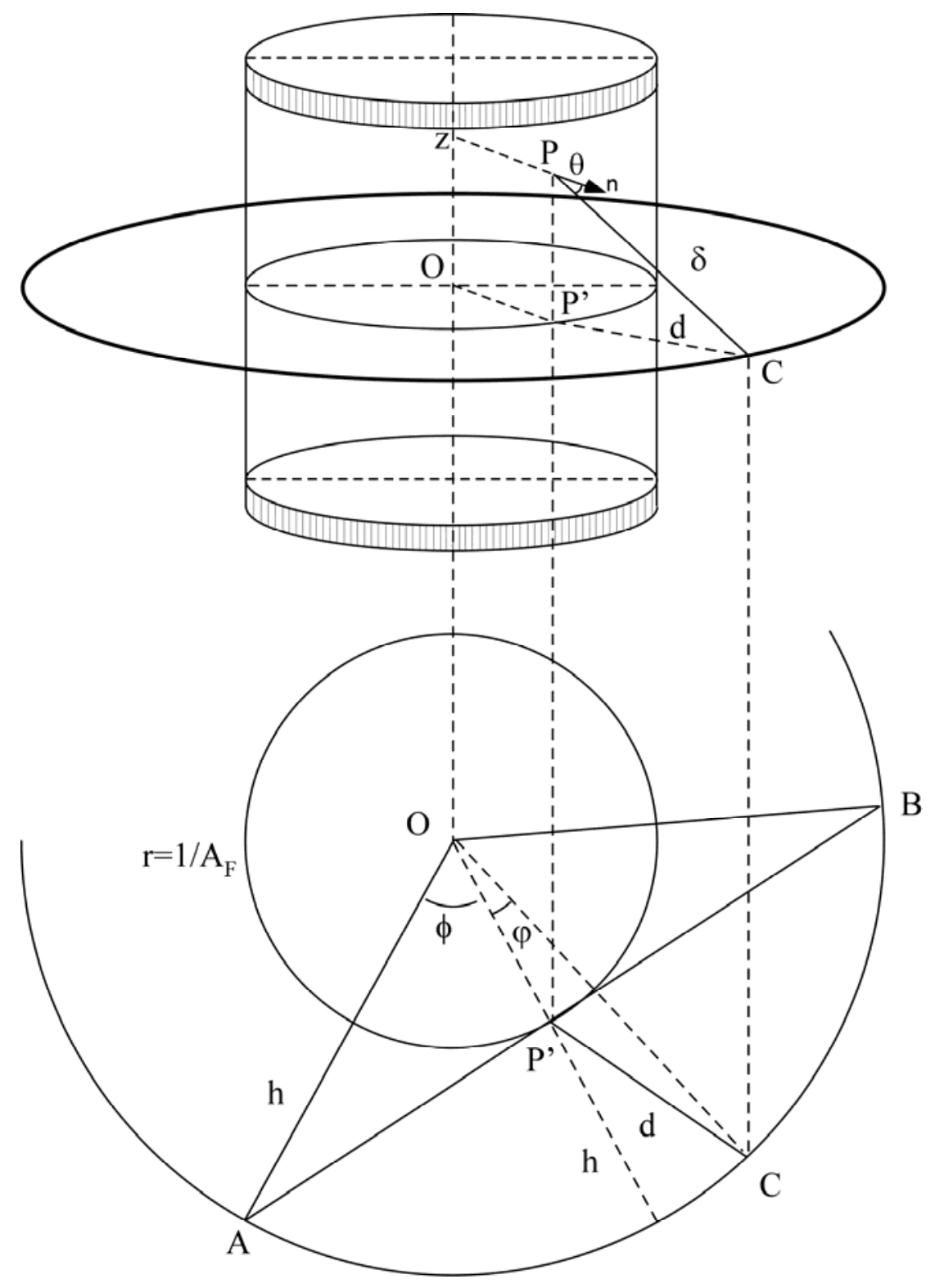

Figure 1: Sketch of the full zone with its ring heater and reference system

\section{B. Basic assumptions}

As shown in Fig. 1, we assume the floating zone to be confined between two coaxial cylindrical disks of diameter $\mathrm{D}$ having an axial distance $2 \mathrm{~L}$. Because these disks are intended to mimic the upper and lower solid-melt interfaces of a real floating zone, they are kept at the same temperature $\bar{T}_{0}$ assumed to represent the typical melting point temperature of the considered material (hereafter, the overbars are used to denote dimensional quantities). As the liquid is heated at the mid-height by an external ring heater, the maximum system temperature, $\bar{T}_{\text {max }}$, is established on the free surface. Following Lappa ${ }^{22}$, the maximum temperature difference achieved for the axi-symmetric (2D) solution assumed as initial condition for the three-dimensional (3D) computations (denoted by $\overline{\Delta T}$ ) is used for the definition of the Marangoni number. 
The liquid is homogeneous and Newtonian. In order to properly capture the physics at work in the experiments by Kudo et al., ${ }^{33}$, the dependence of the kinematic viscosity on the temperature is modeled via the relationship:

$\bar{v}(T)=\bar{v}_{o} \exp \left(c_{1} \frac{\bar{T}_{0}-\bar{T}}{c_{2}+\bar{T}}\right)$

where $\bar{v}_{o}=\bar{v}\left(\bar{T}_{0}\right)$ and $\mathrm{c}_{1}$ and $\mathrm{c}_{2}$ are coefficients depending on the specific liquid and the range of temperatures considered. Here we assume viscous dissipation to be negligible. Moreover, following common practice in the literature, both the liquid density $\bar{\rho}$ and the surface tension $\bar{\sigma}$, characterizing the (volume and surface, respectively) physical forces affecting the considered system, are modeled as:

$\bar{\rho}=\bar{\rho}_{o}-\bar{\beta}_{T}\left(\bar{T}-\bar{T}_{0}\right)$

$\bar{\sigma}=\bar{\sigma}_{o}-\bar{\sigma}_{T}\left(\bar{T}-\bar{T}_{0}\right)$

that is, linear relationships where $\bar{\rho}_{o}$ and $\bar{\sigma}_{o}$ are the respective values for $\bar{T}=\bar{T}_{0}$, while $\bar{\beta}_{T}=-d \bar{\rho} / d \bar{T}$ and $\bar{\sigma}_{T}=-d \bar{\sigma} / d \bar{T}$ are positive coefficients accounting for the decrease of these physical quantities with $T$.

The interface is assumed to be nondeformable and axisymmetric around the z-axis. The geometrical aspect ratio of the full zone $A_{F}$ is defined as $A_{F}=2 L / D$; the aspect ratio of the corresponding half zone (half of the considered full zone) would read accordingly $A_{H}=L / D=A_{F} / 2$.

\section{$\underline{\text { C. Governing equations and boundary conditions }}$}

The governing equations for mass, momentum and energy can be cast in condensed nondimensional conservative form as:

$\underline{\nabla} \cdot \underline{V}=0$

$\frac{\partial \underline{V}}{\partial t}=-\underline{\nabla} p-\underline{\nabla} \cdot[\underline{V} \underline{V}]+\operatorname{Pr}\left[\underline{\nabla}^{2} \underline{V}+2 \underline{\nabla} v \cdot(\underline{\nabla} \underline{V})_{o}^{s}\right]-\operatorname{Pr} \beta_{T}^{*} T \underline{i}_{g}$

$\frac{\partial T}{\partial t}=-\underline{\nabla} \cdot[\underline{V} T]+\nabla^{2} T$

where $\mathrm{V}$ and $\mathrm{p}$ are the non-dimensional velocity and pressure, $\operatorname{Pr}$ is the Prandtl number $\operatorname{Pr}=\bar{v}_{o} / \bar{\alpha}$ where $\bar{\alpha}$ is the thermal diffusivity and $v$ is the nondimensional viscosity $\bar{v} / \bar{v}_{o}$ : 
$v=\exp \left(-c_{1} \frac{T}{\widetilde{c}_{2}+T}\right)$, where $\widetilde{c}_{2}=c_{2}+\bar{T}_{0}$

Moreover, $(\underline{\nabla} \underline{V})_{o}^{s}$ is the strain rate tensor :

$$
(\underline{\nabla} \underline{V})_{o}^{s}=(\underline{\nabla} \underline{V})^{s}-\frac{1}{3}(\underline{\nabla} \cdot \underline{V}) \underline{I}
$$

that for incompressible flow reduces to

$$
(\underline{\nabla} \underline{V})_{o}^{s}=(\underline{\nabla} \underline{V})^{s}=\frac{\underline{\nabla} \underline{V}+\underline{\nabla} \underline{V}^{T}}{2}
$$

The gradient of nondimensional viscosity, in turn, can be rewritten as:

$$
\underline{\nabla} v=\left[\frac{-c_{1} \widetilde{c}_{2}}{\left(\widetilde{c}_{2}+T\right)^{2}}\right] \underline{\nabla} \exp \left(-c_{1} \frac{T}{\widetilde{c}_{2}+T}\right)=v \underline{\nabla} T\left[\frac{-c_{1} \widetilde{c}_{2}}{\left(\widetilde{c}_{2}+T\right)^{2}}\right]
$$

Furthermore,

$$
\beta_{T}^{*}=\frac{\bar{g} \bar{\beta}_{T} L^{3}}{\bar{v}_{0} \bar{\alpha}}
$$

is a dimensional quantity $\left[\mathrm{K}^{-1}\right]$ related to the classical Rayleigh number $\mathrm{Ra}$ by the relationship $\mathrm{Ra}=$ $\beta_{T}^{*}(\overline{\Delta \mathrm{T}})$ with $\underline{\mathrm{i}}_{\mathrm{g}}$ being the unit vector along the direction of gravity.

The above non-dimensional equations result from scaling the cylindrical co-ordinates $(\overline{\mathrm{r}}, \overline{\mathrm{z}})$ by half of the axial distance between the circular disks (L) and the velocity components along the axial, radial and azimuthal directions $\left(\bar{V}_{z}, \bar{V}_{r}, \bar{V}_{\varphi}\right)$ by the energy diffusion velocity $\mathrm{V}_{\alpha}=\bar{\alpha} / \mathrm{L}$; the scales for time and pressure are, respectively, $\mathrm{L}^{2} / \bar{\alpha}$ and $\rho \bar{\alpha}^{2} / \mathrm{L}^{2}$. The temperature (the only primitive variable intentionally left dimensional) is measured with respect the initial temperature $\bar{T}_{0}$.

$T=\left(\bar{T}-\bar{T}_{0}\right)$

No-slip conditions and assigned values of temperature are imposed on the rigid disks at $\mathrm{z}= \pm 1$ :

$$
\begin{array}{ll}
\underline{V}(z=-1, r, \varphi, t)=0 ; \quad T(z=-1, r, \varphi, t)=0 & 0 \leq \mathrm{r} \leq 1 / \mathrm{A}_{\mathrm{F}} ; 0 \leq \varphi \leq 2 \pi \\
\underline{V}(z=1, r, \varphi, t)=0 ; \quad T(z=1, r, \varphi, t)=0 & 0 \leq \mathrm{r} \leq 1 / \mathrm{A}_{\mathrm{F}} ; 0 \leq \varphi \leq 2 \pi
\end{array}
$$


Stream-surface conditions (zero normal velocity) and shear stress balance (the so-called Marangoni condition) are used as relevant boundary conditions on the free surface $\left(r=1 / A_{F},-1 \leq z \leq 1\right.$, $0 \leq \varphi \leq 2 \pi$ ) for kinematic problem closure:

$V_{r}\left(z, r=1 / A_{F}, \varphi, t\right)=0$

$\frac{\partial V_{Z}}{\partial r}\left(z, r=1 / A_{F}, \varphi, t\right)=-\sigma_{T}^{*} \frac{\partial T}{\partial z}\left(z, r=1 / A_{F}, \varphi, t\right)$

$r \frac{\partial V_{\varphi}}{\partial r}\left(z, r=1 / A_{F}, \varphi, t\right)-V_{\varphi}\left(z, r=1 / A_{F}, \varphi, t\right)=-\sigma_{T}^{*} \frac{\partial T}{\partial \varphi}\left(z, r=1 / A_{F}, \varphi, t\right)$

where

$$
\sigma_{T}^{*}=\frac{\bar{\sigma}_{T} L}{\bar{\rho}_{0} \bar{v}_{0} \bar{\alpha}}
$$

is a dimensional quantity $\left[\mathrm{K}^{-1}\right]$ related to the classical Marangoni number Ma by the relationship $\mathrm{Ma}=\sigma_{T}^{*}(\overline{\Delta \mathrm{T}})$. Closure of the resulting mathematical problem finally requires a condition of prescribed heat flux $J(z)$ at the liquid-gas interface:

$$
\frac{\partial T}{\partial r}\left(z, r=1 / A_{F}, \varphi, t\right)=J(z)
$$

Many models have been proposed in literature to simulate surface heating due to a ring heater. It is known that the shape of the effective profile of heat flux at the interface may depend on several factors (see, e.g., Otani et al., ${ }^{36}$; Rivas and Vazquez-Espi ${ }^{37}$ ). For consistency, we adopt the model developed by Lappa ${ }^{22}$, i.e. the radiative flux generated by a ring heater having negligible thickness and positioned around the equatorial plane of the full zone at a fixed distance $h$ from the free surface.

A mathematical expression for such a flux can be derived taking into account the radiative contribution brought to the overall flux by each infinitesimal element pertaining to the ring. According to Fig. 1, this contribution can be cast in compact form as:

$$
j(z)=\left(\frac{1}{L \lambda}\right) \frac{1}{4 \pi} \frac{\xi \bar{Q}}{\delta^{2}} \cos (\vartheta)
$$

where $\overline{\mathrm{Q}}$ is the power supplied to the ring heater, $\delta$ the distance between the points $\mathrm{C}$ (on the ring heater) and $\mathrm{P}$ (on the free surface) and $\theta$ is the angle between $\underline{\mathrm{n}}$ (unit vector orthogonal to the free surface in $\mathrm{P}$ ) and the direction PC. The total flux at the generic point $\mathrm{P}$ on the interface can be finally obtained via straightforward integration of eq. (13), as follows: 


$$
J(z)=\frac{1}{4 \pi}\left(\frac{\xi \bar{Q}}{L \lambda}\right) \int_{-\widetilde{\phi}}^{\widetilde{\phi}} \frac{\cos (\vartheta)}{\delta^{2}} d \varphi
$$

where:

$$
\begin{aligned}
& \delta^{2}=\frac{1}{A_{F}^{2}}\left[\left(1+h A_{F}\right)^{2}+1-2\left(1+h A_{F}\right) \cos \varphi+z^{2} A_{F}^{2}\right] \\
& \cos (\vartheta)=\frac{\left(1+h A_{F}\right) \cos \varphi-1}{\left.\sqrt{\left[\left(1+h A_{F}\right)^{2}+1-2\left(1+h A_{F}\right) \cos \varphi+z^{2} A_{F}^{2}\right.}\right]} \\
& \widetilde{\phi}=\cos ^{-1} \frac{1}{\left(1+h A_{F}\right)}
\end{aligned}
$$

and $\xi$ is the free-surface emissivity.

\section{Numerical method}

\section{A. Projection Method}

These techniques are known under several names: projection method, fractional-step method or pressure-correction method (also simply referred to as primitive-variables approach). This class of methods originates from the studies by Harlow and Welch ${ }^{38}$, Chorin $^{39}$ and Temam ${ }^{40}$. Despite some minor differences, basically, a common feature of all these variants is their "turning around" the relationship between the pressure and the velocity that is established for incompressible fluids (basically, from a purely mathematical point of view, they rely on the Ladyzhenskaya decomposition theorem ${ }^{41}$, which states that any vector function can be split into a part of given divergence and the gradient of a scalar potential). This approach proceeds as a type of fractional step method by first considering a simplified momentum equation and then updating the velocity field using the computed pressure to account for the conservation of mass. More precisely, at each time step, an intermediate velocity field is determined without the knowledge of the correct pressure field, and therefore no incompressibility condition is enforced. The intermediate velocity field is then modified by a second step in which a pressure equation is solved and then the related results are used to produce a divergence-free vector field ${ }^{42}$.

\section{B. Space Discretization and Regularization}

The computational domain has been discretized with a staggered grid, the fluxes and velocities being located at the centers of the faces and, the scalar variables $T$ and $p$ at the center of the cells, respectively. Centered finite-difference schemes with a second-order accuracy have been retained 
for the spatial discretization of both diffusive and convective terms in the momentum equation, whereas a third-order accurate (quick) scheme has been expressly used for the convective contributions appearing in the energy equation (given the delicate role played by temperature disturbances in the considered dynamics). Additional care has been taken in order to damp the spurious oscillations that might be introduced by the well-known issue with the diverging behavior of the surface velocity in proximity to the hot and cold corners. This singularity, potentially affecting the flow at any contact free surface/solid boundary, can jeopardize the physical consistency of the numerical solution and also seriously delay convergence, leading in some circumstances to oscillations that may result in grid-divergence (Bouizi et al., ${ }^{32}$ and references therein).

Usually such a regularization is not necessary for low Prandtl number fluids $(\operatorname{Pr}<<1)$ since in this case the singularity is implicitly bypassed by the filtering properties of the local approximation of the differential operators (in practice, the finite-difference schemes automatically act to regularize, through some filtering mesh-dependent function, the initially singular formulation and the numerical solution converges, asymptotically with the mesh refinement, to the solution of a continuous problem ${ }^{22-25}$ ).

Nevertheless, the explicit regularization becomes a critical requirement for high Prandtl number liquids $(\operatorname{Pr}>1)$ and in particular for large values of the Marangoni number. For these cases grid convergence cannot be obtained or occurs only if very dense (often prohibitive for threedimensional simulations) computational meshes are used. In this case, the regularization approach, rather than relying on the intrinsic filtering properties of the numerical scheme (which in this case are not sufficient), tries to get an explicit regular continuous formulation of the problem through appropriate modeling of the underlying physical regularity ${ }^{32}$.

Here, we introduce the regularizing function as

$\mathfrak{R}_{n}(z)=\left(\frac{1-|z|}{\Delta \xi}\right)^{n} \quad|z| \geq 1-\Delta \xi$

to be inserted in the boundary conditions (10b) and (10c), i.e.:

$$
\frac{\partial \underline{V}}{\partial n}=-M a \Re_{n}(z) \underline{\nabla_{S}} T
$$

where $\mathrm{n}$ is the so-called filtering parameter, to be increased until an asymptotic behavior is attained (to be considered as a proof of the physical relevance of the model). 


\section{Grid Refinement and Validation}

For the case of liquid metals, where no regularization is required, the present code was validated through comparison with other results available in the literature for low-Pr fluids ${ }^{22}$.

For the high-Pr case, where regularization is a critical requirement, our approach was tested by checking its convergence under mesh refinement for both the half- and full-zone configurations.

TABLE I: Comparison with the results of Melnikov et al. (2011), liquid bridge of $\mathrm{NaNO}_{3}$ with aspect ratio $\mathrm{A}_{\mathrm{H}}=0.34, \mathrm{Ma}=20600$ ( $\mathrm{m}$ is the disturbance azimuthal wavenumber, $\Omega$ the associated angular frequency).

\begin{tabular}{lccc}
\hline \hline Ref & $\begin{array}{c}\text { Grid } \\
\left(\mathrm{N}_{\mathrm{z}} \times \mathrm{N}_{\mathrm{r}} \mathrm{xN}\right)\end{array}$ & $\begin{array}{c}\text { Mode } \\
\mathrm{m}\end{array}$ & $\begin{array}{c}\text { Nondimensional angular frequency of } \\
\text { the hydrothermal wave }(\Omega=2 \pi \mathrm{f} / \mathrm{m})\end{array}$ \\
\hline Melnikov et al. (2011) & $40 \times 40 \times 32$ & 3 & 73.3 \\
Present & $32 \times 40 \times 40$ & 3 & 71.4 \\
\hline \hline
\end{tabular}

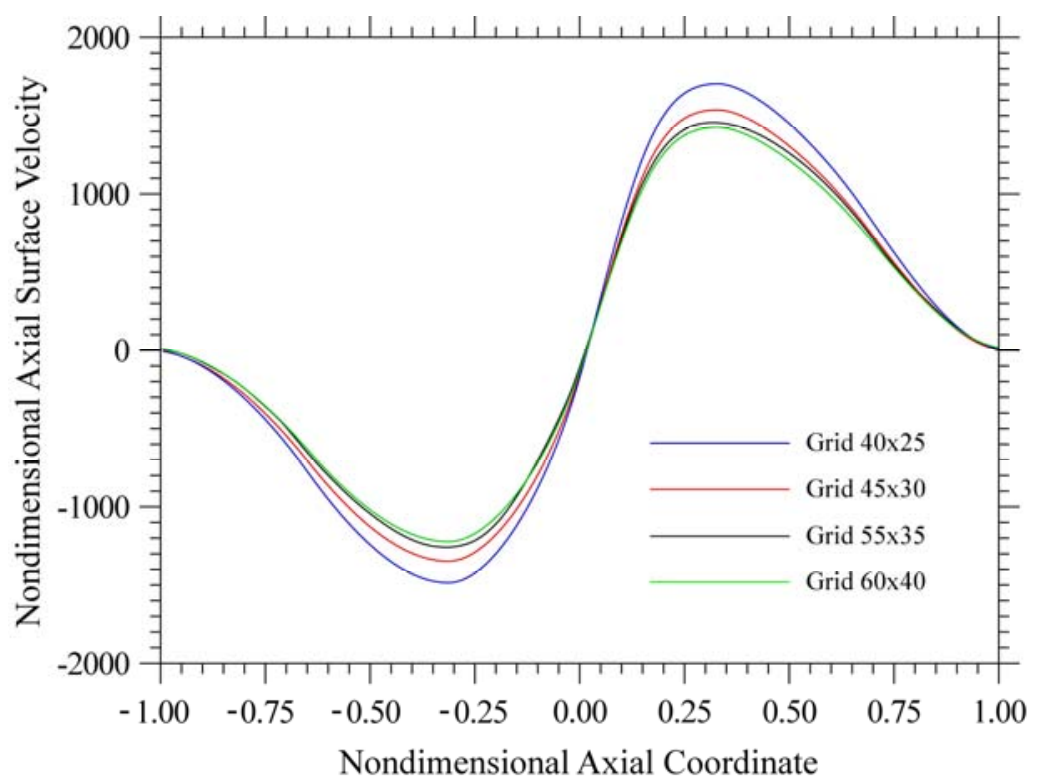

Figure 2: Surface profile of axial velocity (axisymmetric computations) for $\operatorname{Pr}=28.1, \mathrm{~A}_{\mathrm{F}}=0.63$, $\mathrm{Ma} \cong 2.3 \times 10^{4}, \mathrm{Ra} \cong 4.2 \times 10^{3}$, versus mesh resolution $\mathrm{N}_{\mathrm{z}} \times \mathrm{N}_{\mathrm{r}}$.

For the first case the method was verified on the test case reported by Melnikov et al., ${ }^{43}$ (results of the validation study are summarized in Table I, the reader being also referred to Ref. 44 for further details). Results for the full-zone (silicone oil with properties shown in Table II) are summarized in Fig. 2, where the solution mesh-independence is shown in terms of surface velocity profile. We could obtain proper convergence by applying the regularization technique illustrated in Sect. III.B, achieving it for $\mathrm{n}=4$ and $\Delta \xi=0.15$.

By increasing the mesh resolution from $55 \times 35$ to $60 \times 40$, we found the percentage variation experienced by the maximum surface velocity for $A_{F}=0.63$ to be less than $2 \%$, which indicates that 
the former grid may be considered sufficiently dense to guarantee mesh independence. Moreover, following earlier indications provided by Lappa ${ }^{22}$ for comparable values of the full-zone aspect ratio (see table II in that work), we decided to use 70 points in the azimuthal direction.

Following the same procedure, a mesh $65 \times 35 \times 70$ was deemed to be sufficient for $A_{F}=0.88$.

\section{Results}

The properties of the considered fluid (after Kudo et al., ${ }^{33}$ ) are reported in Table II

TABLE II: Properties of the considered $2 \mathrm{cSt}$ Silicone oil at $\bar{T}_{0}=25^{\circ} \mathrm{C}$

\begin{tabular}{ll}
\hline \hline Fluid density $\rho\left[\mathrm{kg} \mathrm{m}^{-3}\right]$ & 873 \\
Kinematic viscosity $v_{0}\left[\mathrm{~m}^{2} \mathrm{~s}^{-1}\right]$ & $2.0 \times 10^{-6}$ \\
$\mathrm{c}_{1}$ coefficient $[-]$ & 5.892 \\
$\mathrm{c}_{2}$ coefficient $\left[{ }^{\circ} \mathrm{C}\right]$ & 273.15 \\
Thermal diffusivity $\alpha\left[\mathrm{m}^{2} \mathrm{~s}^{-1}\right]$ & $7.12 \times 10^{-8}$ \\
Thermal conductivity $\lambda\left[\mathrm{W} \mathrm{m}^{-1} \mathrm{~K}^{-1}\right]$ & 0.11 \\
Prandtl number $[-]$ & 28.1 \\
Thermal expansion coefficient $\beta_{\mathrm{T}}\left[\mathrm{K}^{-1}\right]$ & $1.24 \times 10^{-3}$ \\
Surface tension coefficient $\sigma_{\mathrm{T}}\left[\mathrm{Nm}^{-1} \mathrm{~K}^{-1}\right]$ & $7.15 \times 10^{-5}$ \\
\hline \hline
\end{tabular}

The liquid is a silicone oil with Prandtl number $\operatorname{Pr}=28.1$. Simulations have been performed for a fixed value of the diameter $\mathrm{D}$ of the disks supporting the liquid zone, assumed to be $\mathrm{D}=3.5 \mathrm{~mm}$. However, in order to explore the system sensitivity to the geometrical aspect ratio, two distinct values of $A_{F}$ have been considered, i.e. $A_{F}=0.63$ and $A_{F}=0.88$, as in the experimental study by Kudo et al., ${ }^{33}$. Still following this study, the diameter of the ring heater $D_{H}$ has to be fixed to 4.2 $\mathrm{mm}$ for the considered size of the liquid zone, which means the distance between it and the surface of the liquid is $0.35 \mathrm{~mm}$ only. The resulting values of the parameters $\sigma_{T}^{*}$ and $\beta_{T}^{*}$, defined by eqs. (11) and (7), respectively are summarized in Table III, together with the related value of the nondimensional distance $\mathrm{h}$.

TABLE III: Parameters used for the simulation

\begin{tabular}{lllll}
\hline \hline $\mathrm{A}_{\mathrm{F}}[-]$ & $\mathrm{L}[\mathrm{m}]$ & $\sigma_{T}^{*}\left[\mathrm{~K}^{-1}\right]$ & $\beta_{T}^{*}\left[\mathrm{~K}^{-1}\right]$ & $\mathrm{h}[-]$ \\
\hline 0.63 & $1.1 \times 10^{-3}$ & 634 & 114.5 & 0.318 \\
0.88 & $1.54 \times 10^{-3}$ & 886 & 312 & 0.227 \\
\hline \hline
\end{tabular}

By application of a radiative heat flux (eq. 14), the heat transport is maximum in the surface region of the liquid zone and changes the driving temperature gradients in the surface by itself (i.e. via the 
Marangoni stress acting on the free interface; as an example, the relationship between $(\overline{\Delta T})$ and the quantity $\xi \bar{Q}$ as provided by axisymmetric simulations is shown in Fig. 3 for $\mathrm{A}_{\mathrm{F}}=0.63$ and $\mathrm{A}_{\mathrm{F}}=0.88$ ). As already explained in Sect. IIB, such a temperature difference is used as a reference value for the evaluation of the non-dimensional Marangoni and Rayleigh numbers $\left(\mathrm{Ma}=\sigma_{T}^{*}(\overline{\Delta T})\right.$ and $\mathrm{Ra}=\beta_{T}^{*}$ $(\overline{\Delta T})$, respectively).
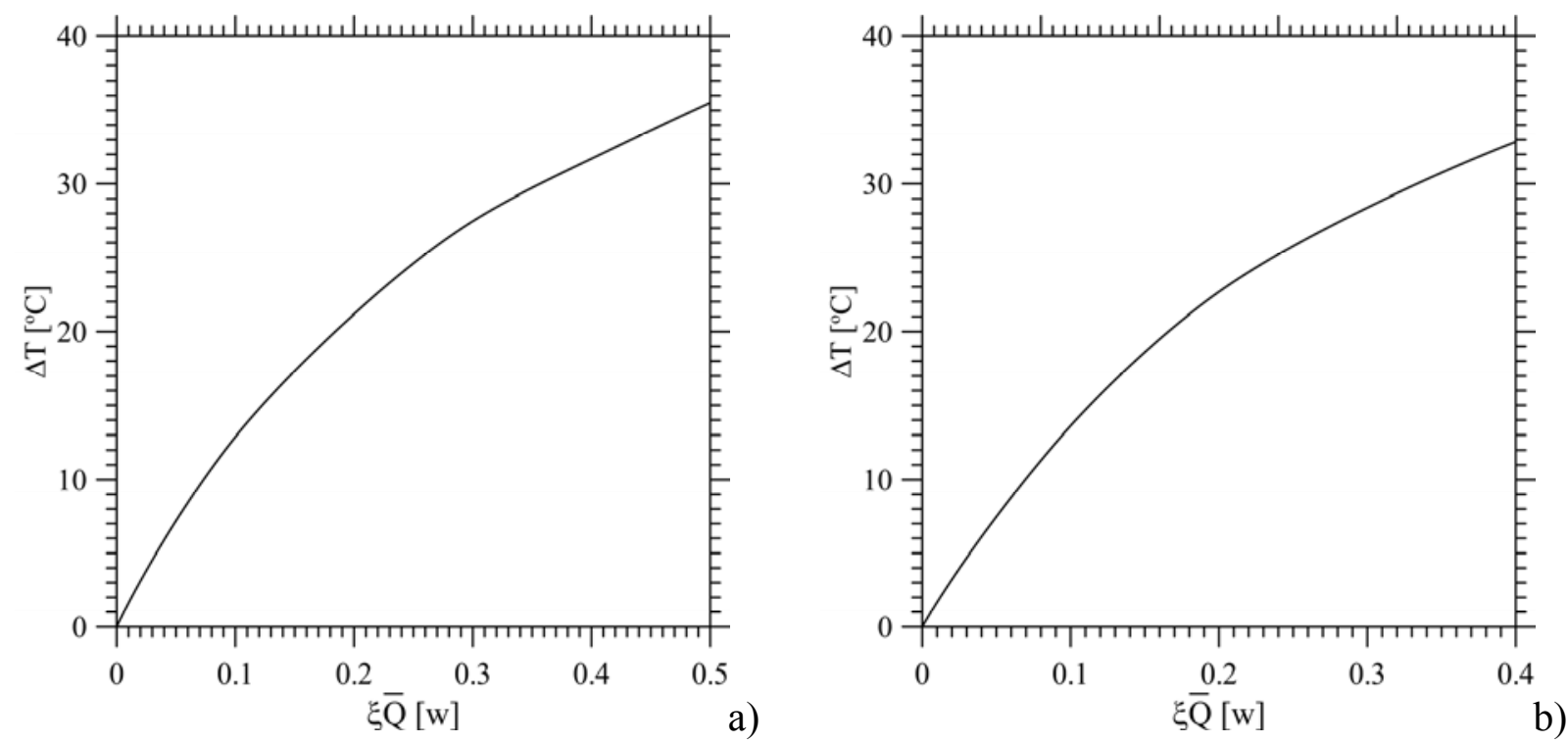

Figure 3: Temperature difference as a function of $\xi \bar{Q}$ for $\operatorname{Pr}=28.1$ (axisymmetric numerical simulations): a) $\left.\mathrm{A}_{\mathrm{F}}=0.63, \mathrm{~b}\right) \mathrm{A}_{\mathrm{F}}=0.88$.

\section{A. The structure of the basic flow and the onset of oscillatory flow}

Prior to embarking into an exhaustive description of the 3D results, it is worth providing some initial and fundamental information about the structure of the basic (axisymmetric steady) flow field (which will prove very useful later when discussing the general features of the supercritical state).

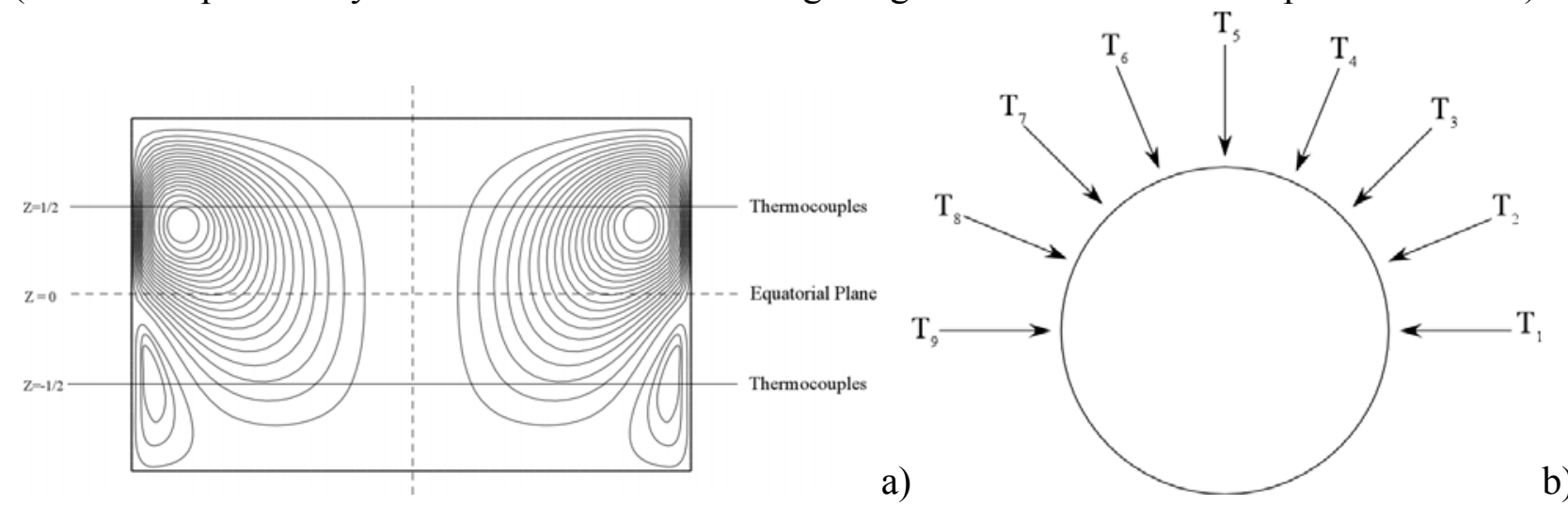

Figure 4: Sketch: a) typical flow in the full zone under normal gravity conditions; b) thermocouples distribution along the circumferential extension at a fixed axial position $(\mathrm{z}=1 / 2$ and $\mathrm{z}=-1.2$ for the upper and lower half of the floating zone, respectively). 
Along these lines, we start from the simple observation that due to the presence of gravity, which breaks the ideal system symmetry with respect to the equatorial plane, the basic state is no longer symmetric with respect to $\mathrm{z}=0$ (as it would be in microgravity conditions ${ }^{22}$ ).

Indeed, two clearly distinguishable toroidal rolls are present in the floating zone, each being driven by the temperature gradient established between the area of maximum heating and one of the supporting disks. Despite the intrinsic symmetry of the geometry and thermal and kinematic boundary conditions, these rolls are strongly asymmetric (Fig. 4a).

The nature of this asymmetry can be explained on the basis of simple rational arguments, among them, the delicate interplay established between buoyancy effects and thermocapillarity that is established in each half of the floating zone. Because, roughly speaking, the upper half might be thought of as a system being heated from below, while an opposite situation might be imagined for the lower half, the reader will easily realize that buoyancy will be acting as a force supporting surface Marangoni flow and opposing to it according to whether it is located above and below the equatorial plane, respectively. Instructive examples along these lines can be found for instance in the earlier literature published for the simplified half-zone case (the classical liquid bridge, see, e.g., Frank and Schwabe ${ }^{1}$ ).

Similar effects are at the root of the pattern-forming mechanism in the complete floating zone. The flow consists essentially of two superposed toroidal rolls, with the roll located in the upper half (where Marangoni and buoyant flows are concurrent) being stronger and protruding to a large extent into the lower half; by contrast, the (weaker) bottom roll takes an axially stretched shape and is confined to a region of relatively small radial extension located in proximity to the liquid/gas interface (Fig. 4a). Each of these convection rolls is bounded by a wall from one side and it is free to interact in nonlinear way with the opposite convection roll from the other side ${ }^{23}$.

These relatively simple considerations reinforce the idea that, quantitatively speaking, earlier results for the half zone might be of scarce relevance to the present case as the complete structure of the convective field would be required in principle to account for non-linearities and other complex effects arising from the mutual interaction of the two superposed toroidal vortices.

In facts, this conjecture is confirmed by the value of the critical Marangoni number provided by the present analysis. This value is much smaller $(\cong 50 \%)$ than that reported in the past for liquid bridges in equivalent conditions. Following common practice in the literature, we determined it by extrapolating to zero the disturbance growth rate obtained by monitoring the time history of the azimuthal disturbances for different values of the Marangoni number. Through this procedure, in particular, we obtained: $\mathrm{Ma}_{\mathrm{cr}} \cong 8.7 \times 10^{3}$ and $\mathrm{Ma}_{\mathrm{cr}} \cong 1.46 \times 10^{4}$ for $\mathrm{A}_{\mathrm{F}}=0.63$ and $\mathrm{A}_{\mathrm{F}}=0.88$, respectively.

Such values are in reasonable agreement with those reported experimentally by Kudo et al., ${ }^{33}$ namely $\mathrm{Ma}_{\mathrm{cr}} \cong 7 . \times 10^{3}$ for $\mathrm{A}_{\mathrm{F}}=0.63$ and $\mathrm{Ma}_{\mathrm{cr}} \cong 1.2 \times 10^{4} \mathrm{~A}_{\mathrm{F}}=0.88$, the observed $\cong 20 \%$ difference being probably due to the unavoidable differences between experiments and numerical simulations (despite the effort we put in reproducing at the best of our capabilities the effect of a ring heater, it must be taken into account that in our model the thickness of the wire was assumed to be negligible, which is obviously an idealization). Another source of difference could be related to the weak, but 
not negligible, deformation displayed by the surface shape during the experiments (this will be the subject of a future paper entirely devoted to model such aspects).

Interestingly, by comparison of numerical simulations carried out turning off and on, respectively, the dependence of viscosity on temperature, we could discern that assuming a constant viscosity would even determine an increase in the difference between the current values of the critical Marangoni number and those obtained experimentally (the numerically determined values at constant viscosity being $\mathrm{Ma}_{\mathrm{cr}} \cong 1.087 \times 10^{4}$ for $\mathrm{A}_{\mathrm{F}}=0.63$ and $\mathrm{Ma}_{\mathrm{cr}} \cong 1.73 \times 10^{4} \mathrm{~A}_{\mathrm{F}}=0.88$ ), which justifies our decision to consider such a dependence.

\section{$\underline{\text { B. Flow spatial structure }}$}

Still following Kudo et al., ${ }^{33}$ we have explored a relatively range of supercritical conditions: $0<\varepsilon \leq 1.5$ ( $\varepsilon$ defined in the classical way, i.e. as $\left(\mathrm{Ma}-\mathrm{Ma}_{\mathrm{cr}}\right) / \mathrm{Ma}_{\mathrm{cr}}$ ).

Given the peculiarity of the considered problem, insights into the considered dynamics have been sought through mathematical modeling and numerical solutions reflecting a combination of concepts, points of view and principles, complementing each other and allowing "capturing" and describing the physics of the problem under different perspectives.

Along these lines, as an example, 1) our direct observation of the maps of azimuthal velocity in cross-sections perpendicular to the zone axis has been instrumental in identifying the dominant azimuthal wavenumber and its odd or even symmetry, while 2) we have used the phase shifts related to signals provided by numerical probes at the same axial and radial coordinates but different azimuthal locations to detect the prevailing spatiotemporal oscillatory mode (Fig. 4b, the reader being referred to Sect. $\mathrm{V}$ for some additional details into the criteria used to identify the prevailing waveform and related transitions).

By such a strategy we could discern that while for relatively small values of $\varepsilon$, the flow shows the classical evolutionary process with a disturbance having well-defined wavenumber growing in time until its amplitude is saturated and a stable oscillatory pattern with well-defined frequency is established (just as it occurs when liquid bridges are used), with the full zone it is sufficient to slightly increase $\varepsilon$ above 0.3 to get very unusual scenarios. Some snapshots along these lines are shown in Figs. $5-8$ (for brevity results are presented for $\mathrm{A}_{\mathrm{F}}=0.63$ and $\mathrm{Ma} \cong 2.3 \times 10^{4}$, the dynamics obtained for the other considered value of the aspect ratio and of $\varepsilon$ being very similar from a qualitative point of view; we concentrate on such a value of the Marangoni number to allow direct comparison with earlier results obtained for the liquid bridge at comparable values of $\operatorname{Pr}$ and Ma, Lappa et al., ${ }^{35}$ ).

Careful analysis of these plots, especially those showing the distribution of the azimuthal velocity in cross-sections perpendicular to the $\mathrm{z}$ axis, indeed reveals almost immediately that the flow displays an increased degree of complexity with respect to earlier half-zone based results. An immediate and unambiguous identification of the azimuthal wavenumber is indeed rather difficult. Different disturbances with distinct spatial structure seem to overlap in space and alternate in time. 
We found the best approach to deal with such intricacies to be based on the direct observation of the multicellular structures emerging in selected cross sections of the two opposing rolls and on the examination of the spatial morphology and topology of some selected disturbance isosurfaces.

Indeed, by inspection of Figs. 5-8, the reader will immediately realize that the "multiplicity" of the involved azimuthal modes is $\mathrm{N}=4$, as the azimuthal wavenumbers $\mathrm{m}=1,2,3$ and 4 can be identified in isolated or combined form. Such figures also provide interesting information of the relative amplitude of disturbances in the two rolls. Indeed, by denoting with $f_{(\text {down })}(r, z, t)$ and $f_{(u p) m}(r, z, t)$ the amplitude of the generic disturbance with azimuthal wavenumber $\mathrm{m}$ in the lower and upper roll, respectively, cross-comparison of sections at $\mathrm{z}=1 / 2$ and $\mathrm{z}=-1 / 2$ leads to the general conclusion that for a given $\mathrm{z}>0, f_{(u p) m}(r, z, t) \neq f_{(\text {down }) m}(r,-z, t)$, with

$\left|f_{(\text {down }) m}(r,-z, t)\right| \leq\left|f_{(u p) m}(r, z, t)\right|$

which in some circumstances and for some specific modes (Figs. 5 and 6) even reduces to

$f_{(\text {down }) m}(r,-z, t) \cong 0$

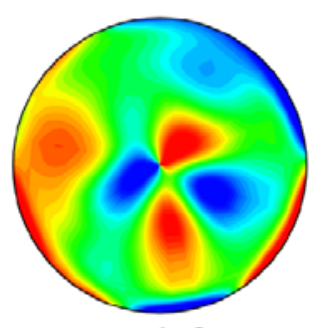

$\mathrm{m}=1+2$

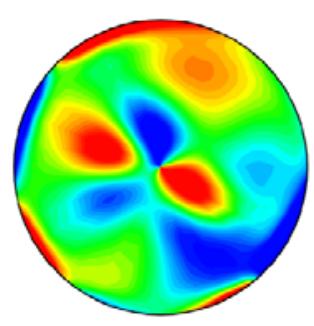

$\mathrm{m}=2+3$

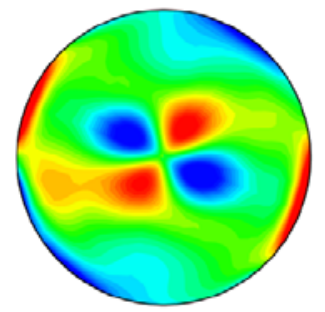

$\mathrm{m}=2$

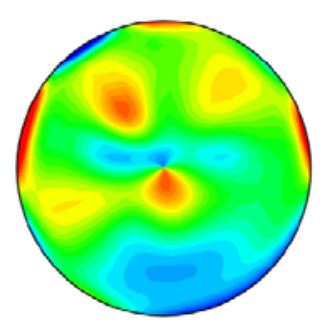

$\mathrm{m}=1+4$

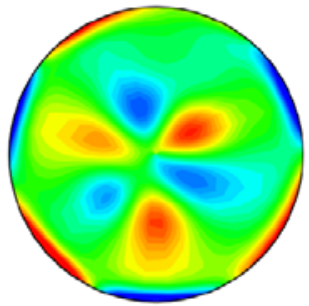

$\mathrm{m}=3$

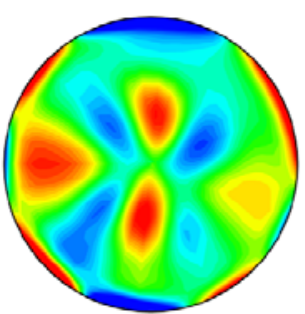

$\mathrm{m}=2+4$

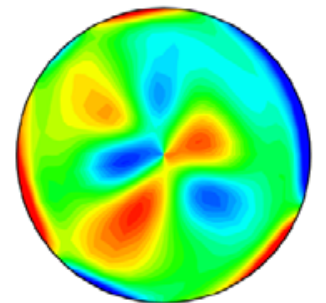

$\mathrm{m}=1+3$

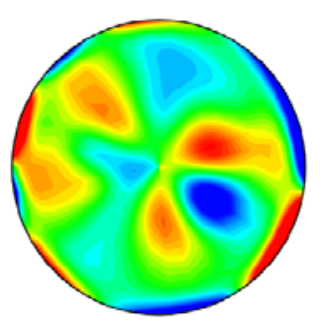

$\mathrm{m}=3+4$

Figure 5: Snapshots of azimuthal velocity distribution at different times in cross-sections perpendicular to the $\mathrm{z}$ axis $\left(\mathrm{z}=1 / 2\right.$, upper half, $\left.\operatorname{Pr}=28.1, \mathrm{~A}_{\mathrm{F}}=0.63, \mathrm{Ma} \cong 2.3 \times 10^{4}, \operatorname{Ra} \cong 4.2 \times 10^{3}\right)$.

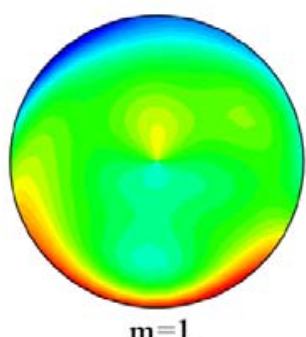

$\mathrm{m}=1$

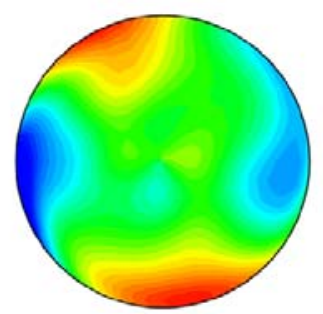

$\mathrm{m}=2$

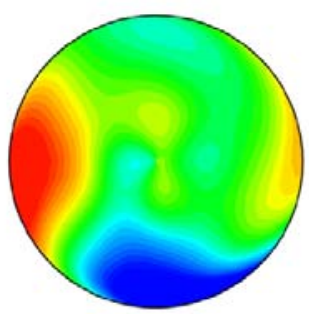

$\mathrm{m}=1+2$

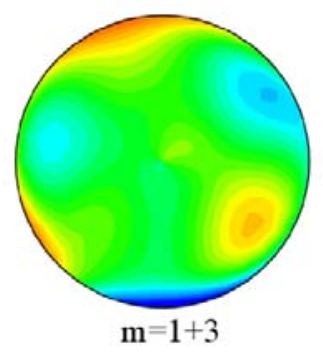

$\mathrm{m}=1+3$

Figure 6: Snapshots of azimuthal velocity distribution at different times in cross-sections perpendicular to the $\mathrm{z}$ axis $\left(\mathrm{z}=-1 / 2\right.$, lower half, $\left.\operatorname{Pr}=28.1, \mathrm{~A}_{\mathrm{F}}=0.63, \mathrm{Ma} \cong 2.3 \times 10^{4}, \mathrm{Ra} \cong 4.2 \times 10^{3}\right)$. 


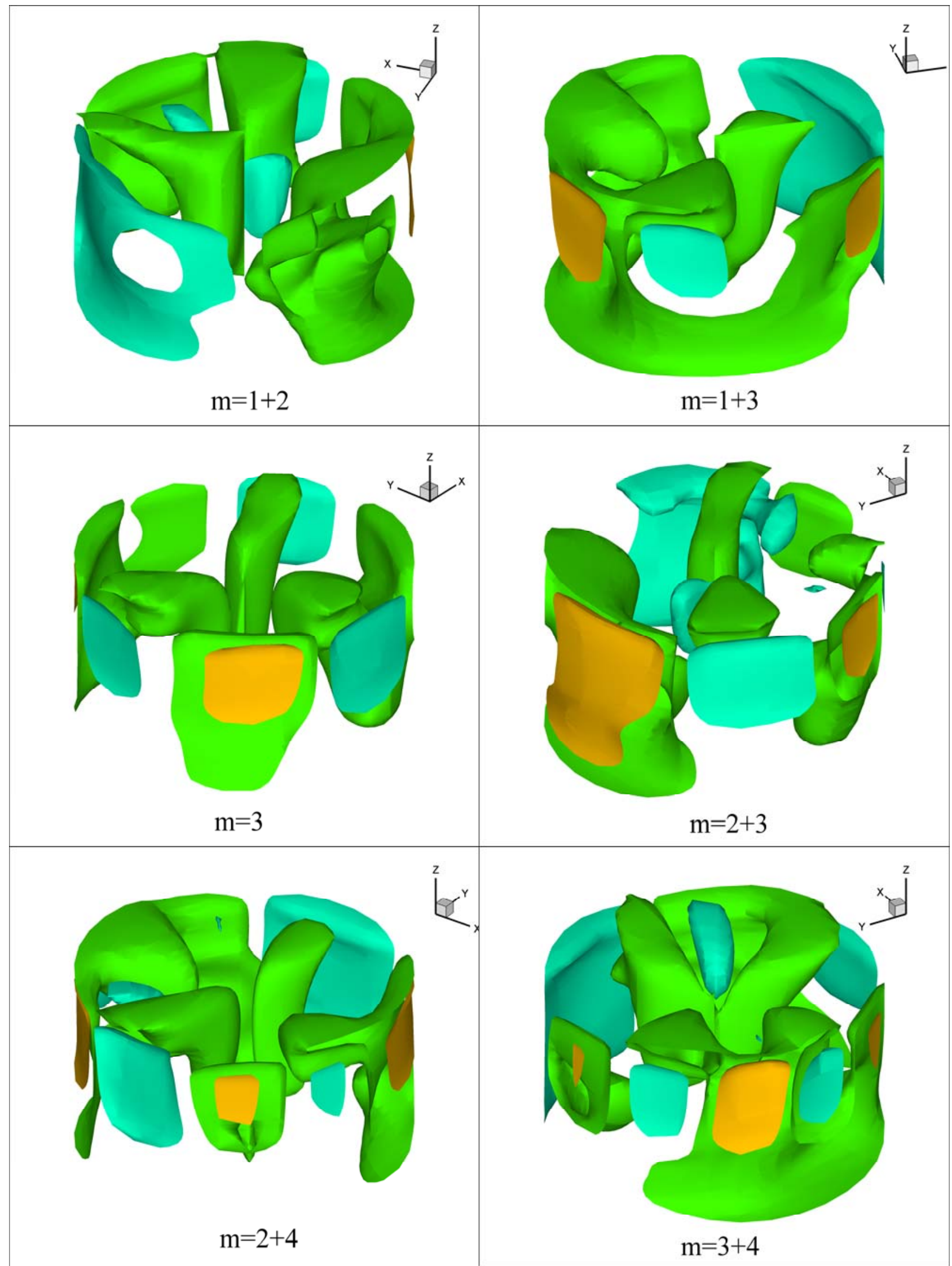

Figure 7: Snapshots of isosurfaces of azimuthal velocity at different times $\left(A_{F}=0.63\right)$. The isosurfaces correspond to three distinct values of the nondimensional azimuthal velocity $(-7 \times 10$, $2 \times 10,1.1 \times 10)$. 
The most striking finding or feature is that, while a relatively rich set of of azimuthal wavenumbers is allowed for the upper roll ( $\mathrm{m}=1,2,3,4$ and related combinations or "hybrid states" as explained before), the variety of such modes is dramatically reduced when the lower roll is examined (there, only disturbances with $\mathrm{m}=1$ and $\mathrm{m}=2$ in disjoint or combined form seem to be a persistent feature of the dynamics, with some occasional manifestations of the $\mathrm{m}=3$ mode). This conclusion is also supported by the results shown in Fig. 7.

It can be clearly seen there that the distribution of isosurfaces (showing the space distribution of azimuthal velocity) undergoes a dramatic change in the lower half of the domain. Most of disturbance "nodes", clearly visible in the form of separated "pillows" with different colors distributed along the circumferential direction above the equatorial plane, are indeed replaced by "blank regions" (even leading to a completely patternless state in some circumstances) if one looks at their distribution under this plane. This is especially true for the disturbances with $\mathrm{m}=3$ and $\mathrm{m}=4$ (the corresponding isosurfaces or "pillows" are no longer visible in the lower half), which indicates that eq. (17b) (which we have introduced before without providing, however, too many details), effectively holds and it is satisfied essentially in the large wavenumber part of the spectrum of disturbances.

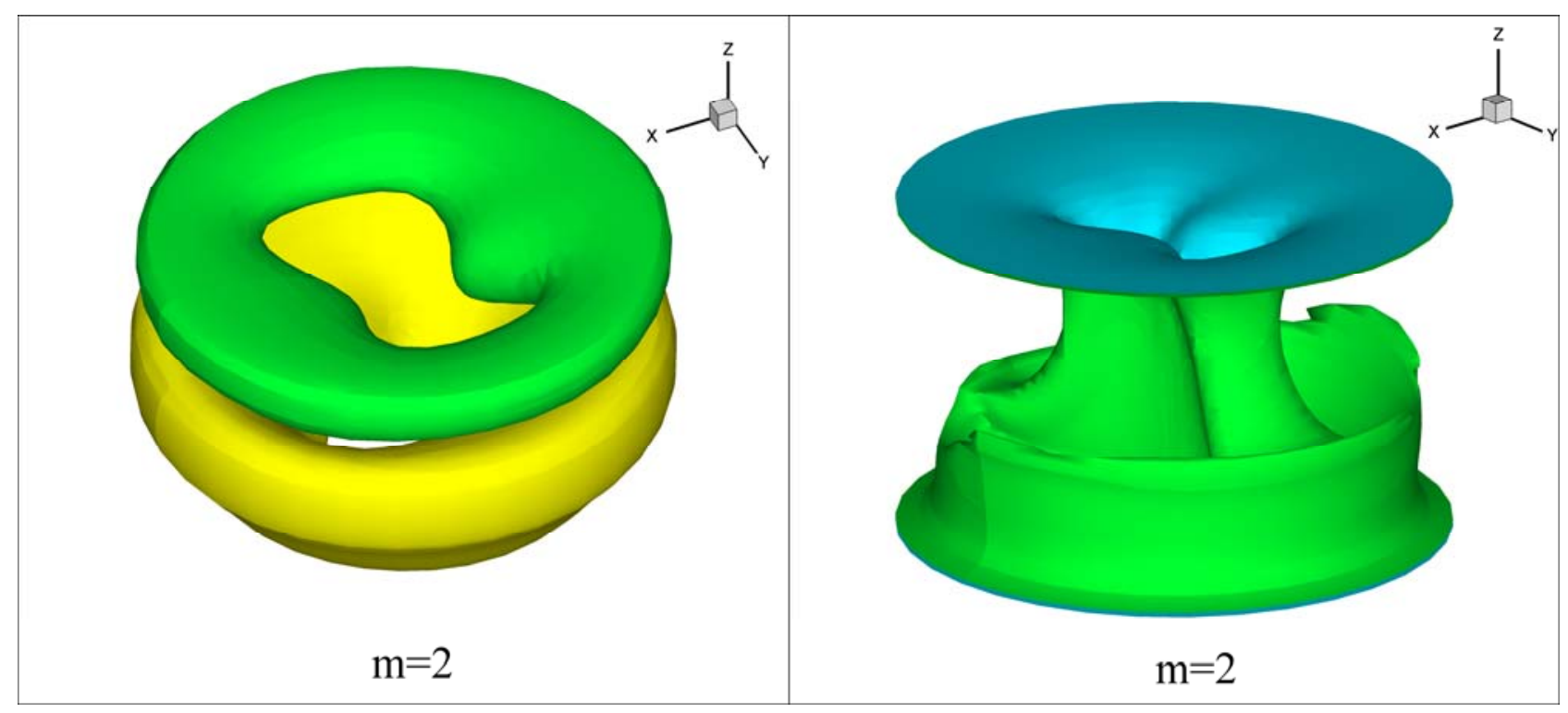




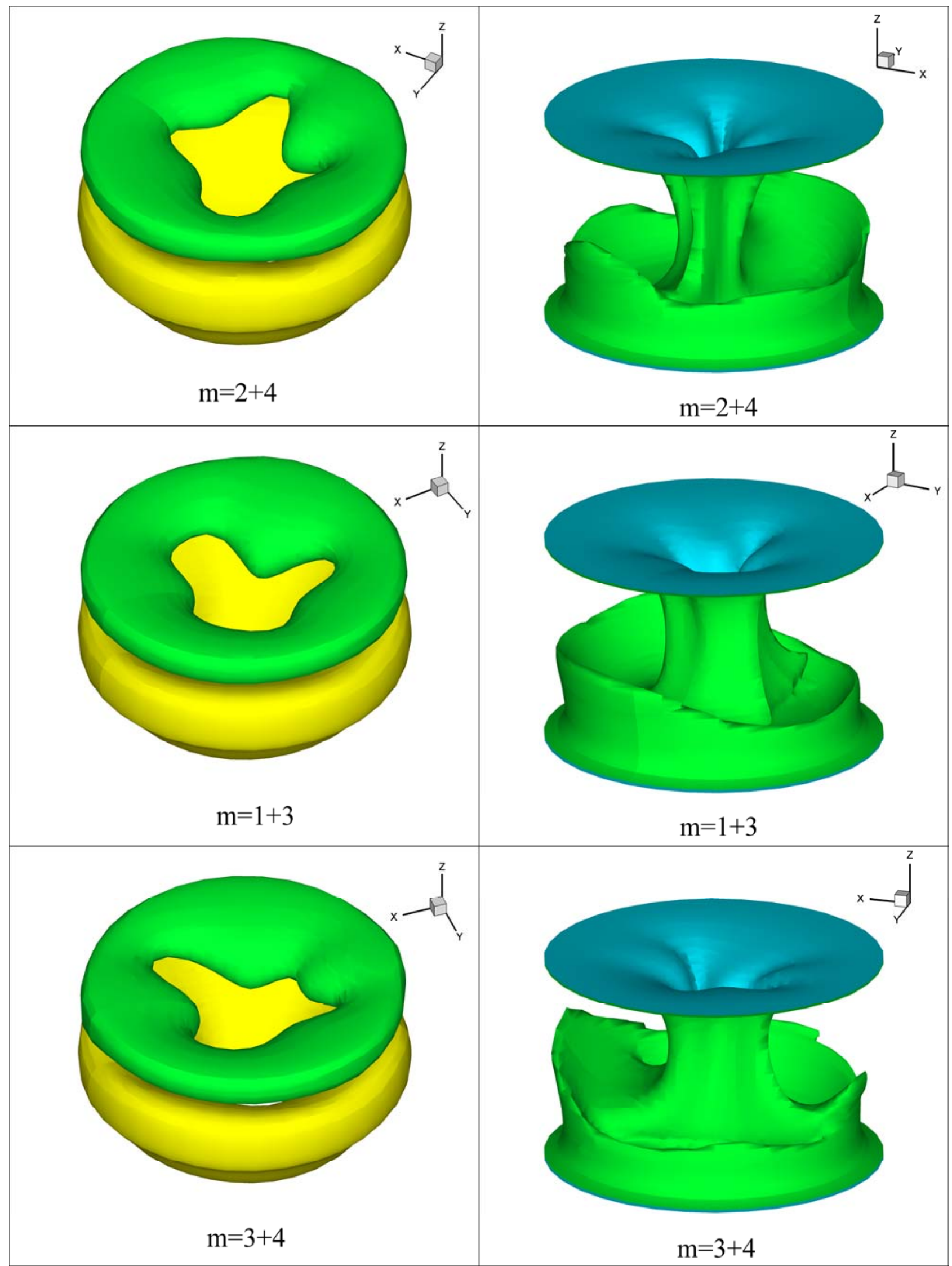

Figure 8: Snapshots of isosurfaces of radial velocity (left) and temperature (right) distributions at different times $\left(A_{F}=0.63\right)$. The values corresponding to the isosurfaces are $\left(-2 \times 10^{2}\right.$ and $\left.10^{2}\right)$ for the radial velocity and (11 and 22) for the temperature, respectively. 


\section{Temporal Dynamics}

Having finished a description of the flow from a spatial perspective, it is now instructive to attempt an interpretation of the related temporal dynamics on the basis of past "models", which so much success have enjoyed in the framework of analyses devoted to the liquid bridge (namely, the socalled "standing wave" and "travelling wave").

Although such waveforms have been successfully applied to describe the dynamics of supercritical Marangoni flow in a variety of circumstances, a critical assessment of the the present numerical results leads to the conclusion that a direct application of them to the present case is not so straightforward as one would imagine. The numerically simulated dynamics are indeed rather irregular, even apparently erratic in some circumstances.

A more accurate inspection of the stages of evolution displayed by the system, however, reveals that some timeframes of limited extent can be effectively identified in which the system may be said to partially fit the just discussed theoretical behaviors (the reader being referred to the quite regular waveforms in Figs. 9-12).
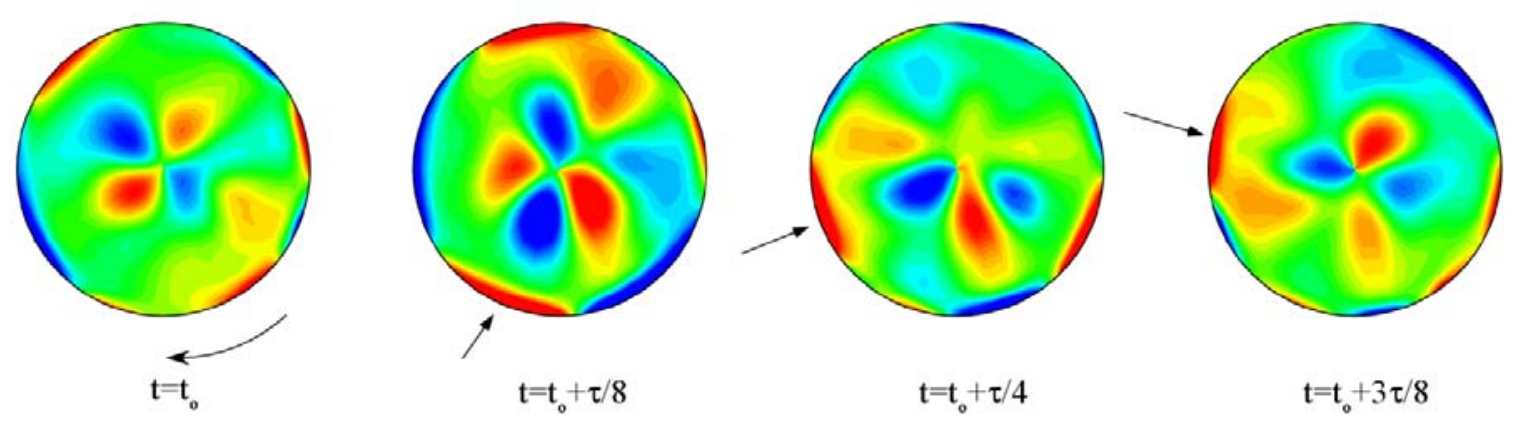

Figure 9: Four snapshots of azimuthal velocity distribution at $z=1 / 2$ (upper half) evenly spaced in time showing the propagation of a disturbance in the clockwise direction (Clockwise traveling disturbance originating at $\mathrm{t}_{\mathrm{o}} \cong 46.7 \mathrm{~s}, \tau \cong 0.7 \mathrm{~s}, \mathrm{~A}_{\mathrm{F}}=0.63$ ).

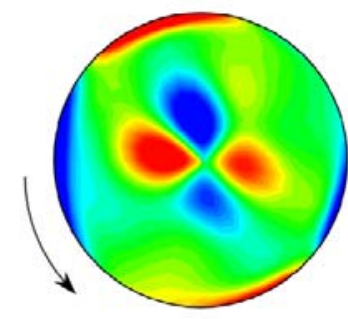

$\mathrm{t}=\mathrm{t}_{\mathrm{o}}$

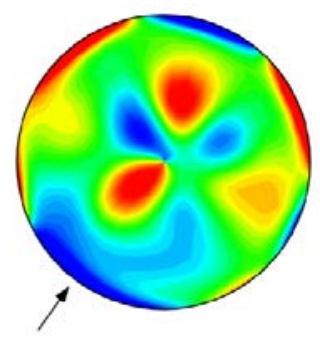

$\mathrm{t}=\mathrm{t}_{\mathrm{o}}+\tau / 8$

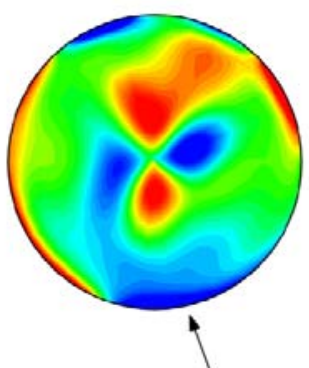

$\mathrm{t}=\mathrm{t}_{\mathrm{o}}+\tau / 4$

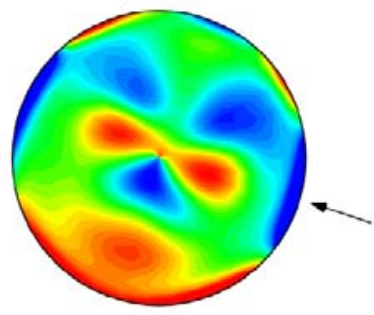

$\mathrm{t}=\mathrm{t}_{\mathrm{o}}+3 \tau / 8$

Figure 10: Four snapshots of azimuthal velocity distribution at $z=1 / 2$ (upper half) evenly spaced in time showing the propagation of a disturbance in the anticlockwise direction (Anticlockwise traveling disturbance originating at $\mathrm{t}_{0} \cong 51.6 \mathrm{~s}, \tau \cong 0.7 \mathrm{~s}, \mathrm{~A}_{\mathrm{F}}=0.63$ ). 

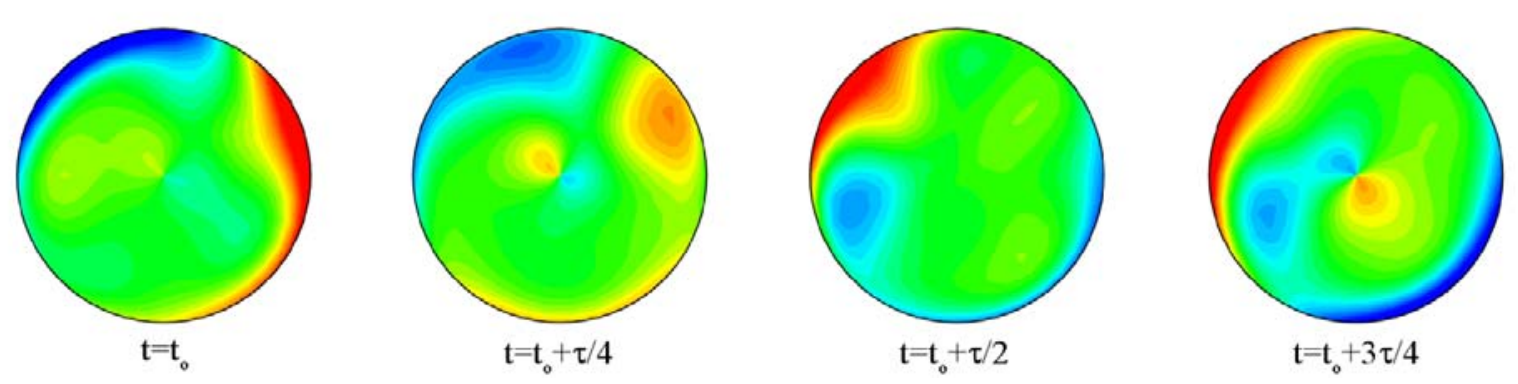

Figure 11: Four snapshots of azimuthal velocity distribution at $z=-1 / 2$ (lower half) evenly spaced in time showing a pulsating regime with wavenumber $\mathrm{m}=1$ (standing wave originating at $\mathrm{t}_{\mathrm{o}} \cong 48.4 \mathrm{~s}$, $\left.\tau \cong 1.1 \mathrm{~s}, \mathrm{~A}_{\mathrm{F}}=0.63\right)$.
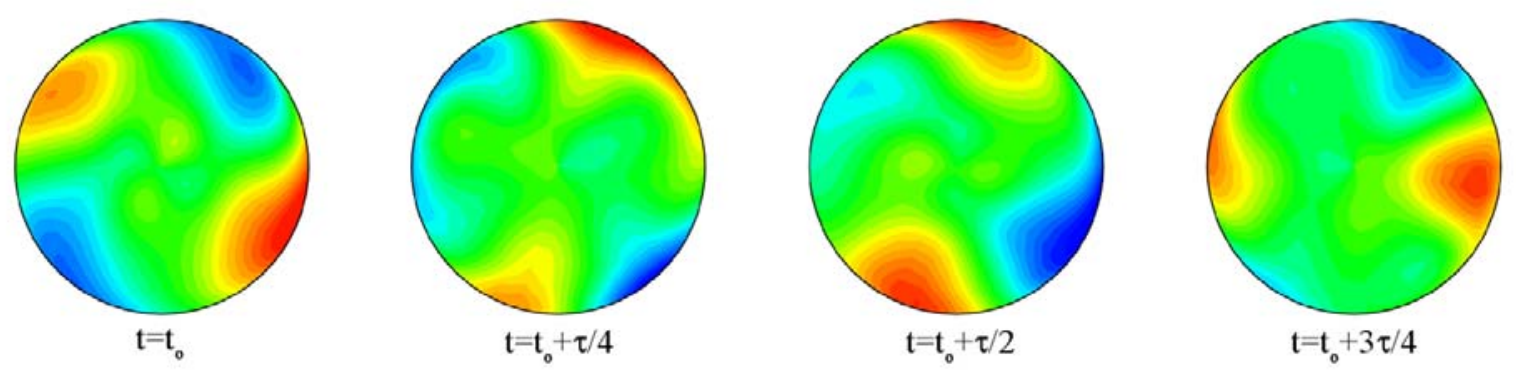

Figure 12: Four snapshots of azimuthal velocity distribution at $z=-1 / 2$ (lower half) evenly spaced in time showing a pulsating regime with wavenumber $\mathrm{m}=2$ (standing wave originating at $\mathrm{t}_{0} \cong 45.9 \mathrm{~s}$, $\tau \cong 0.7 \mathrm{~s}, \mathrm{~A}_{\mathrm{F}}=0.63$ ).

As an example, we could discern in the overall sequence of snapshots provided by the numerical simulations a disturbance travelling in the clockwise direction in the upper half $(\mathrm{z}=1 / 2)$ of the full zone, starting at a time $\mathrm{t}_{\mathrm{o}} \cong 46.7$ and ending at $\mathrm{t} \cong 47.4$.

A similar (clearly recognizable) behavior could be detected after approximately 5 seconds of simulated dimensional time $\left(\mathrm{t}_{0} \cong 51.6\right)$. The disturbance, however, was found to to propagate in the opposite direction in this second instance (i.e. in the anticlockwise sense).

Some timeframes clearly displaying a pulsating scenario could be identified as well, especially when looking at the sequence of snapshots related to the lower half (as witnessed by the sequences reported in Figs. 11 and 12 showing recognizable standing waves with dominant wavenumber $\mathrm{m}=1$ or $\mathrm{m}=2$, respectively). These observable behaviors were not exclusive of a given roll, nor were they truly progressive in time. We observed frequent transitions from one waveform to the other and vice versa in both superposed rolls.

\section{Discussion}

\section{A. Past Studies}

Over recent years, other notable cases representing a "deviation" with respect to the standard occurrence of known (and expected) modes have been detected and studied for different kinds of 
convection $^{45-48}$. The experiments by Kudo et al., ${ }^{33}$, supported by the present numerical results, however, are a first example of exotic dynamics reported for Marangoni flow in floating zones. Earlier studies focusing on the simplified liquid bridge problem revealed great sensitivity to the aspect ratio and well distinct thresholds for different modes in the space of parameters. Furthermore, for not too large supercritical conditions, these studies have always shown patterns with a regular behavior in time with a fixed (well-defined) value of the azimuthal wavenumber.

\section{B. The Mental Divisibility of the Flow and the Disturbances Selection Mechanism}

In this section we will show how the key to interpret the present numerical results lies essentially in the mental divisibility of the flow into two distinct overlying circulation systems with different intensity and morphological properties.

Along these lines, it is convenient to start the discussion by recalling that, if these rolls were isolated, on the basis of past findings for the classical liquid bridge, owing to their different geometrical properties they should undergo rather different instability behaviors. Some additional interesting information could be also drawn on the basis of the earlier works by Lappa ${ }^{22-25}$. Though those studies were concerned with a low-Pr fluid (silicon melt), they could demonstrate that when a two-roll configuration is considered, the interaction of the two opposing vortices can lead to a significant lowering of the instability threshold with respect to the liquid-bridge case and ensuing significant increase in the complexity of the resulting supercritical flow. A similar complexity has been highlighted by Gelfgat et al., ${ }^{49}$, who examined a cylindrical configuration relevant to the socalled vertical Bridgman crystal growth method (melt undergoing buoyancy convection). These authors identified the potential complex interaction of several azimuthal modes which become critical at relatively close values of the Rayleigh.

Evidence supports the idea that similar mechanisms are at play for the configuration considered here. It can be indeed argued that due to the aforementioned interplay between the two opposing circulation systems, which acts expanding the set of potential disturbances and lowering accordingly the instability threshold (as already known for the case of liquid metals), several azimuthal modes are excited at the same time for the considered value of the Marangoni number. In turn, the variety of modes and their diversification according to the roll (upper or lower), can be interpreted on the basis of the different geometrical properties of such convective systems and the different role taken by gravity according to whether the considered region is located over or under the equatorial plane.

\section{An Interpretation of the Spatio-temporal Dynamics}

The interpretation of the spatio-temporal behavior requires a much more elaborated discussion.

For the liquid-bridge (half zone) problem and high values of the Prandtl number (see, e.g., Shevtsova et al., ${ }^{5}$ ), as already discussed to a certain extent in Sect. IV.C., typical oscillatory 
waveforms are known to be standing waves or travelling waves. Possible smooth transitions from the pulsating to the travelling regime have been observed by different authors for increasing values of the Marangoni number or for sufficiently long observation times (for slightly supercritical conditions ${ }^{35}$ ). More rarely, transitions from the travelling wave to the standing wave have been obtained numerically (Melnikov, 2013, private communication), but a continuous (though irregular) switching from one another has never been observed in the framework of past studies dealing with the liquid bridge.

We have already discussed (Sect. IV.C) the possibility to identify some short timeframes in which the classical models of standing wave or the travelling wave seem to be effectively applicable to describe the present flow. Nevertheless, a more in depth analysis of such dynamics calls for a different (complementary and less heuristic) approach based on the analysis of the disturbances propagating along the circumferential direction, their superposition and the resulting behavior in terms of signals provided by probes located at specific positions in the fluid.

In the remainder of this section, in particular, we will base most of our arguments on the wellknown possibility to represent the supercritical state of Marangoni flows in liquid columns in terms of superposition of disturbances traveling along the clockwise and anticlockwise azimuthal direction. Indeed, one of the most interesting outcomes of past theoretical efforts for the liquidbridge problem was the recognition for the effective dynamics and related transitional stages occurring in real experiments to be largely determined by the relative amplitude with which these counter-propagating waves interact (equal or different amplitudes, Kuhlmann and Rath $^{50}$ ).

Modeling each disturbance as a wave having an amplitude $\mathrm{B}(\mathrm{r}, \mathrm{z})$, phase $\mathrm{G}(\mathrm{r}, \mathrm{z})$ and angular frequency $\omega=2 \pi f$ of the type $F_{ \pm}=B(r, z) \exp \{i[ \pm m \varphi-\omega t+G(r, z)]\}$, Kuhlmann and Rath ${ }^{50}$ were able to show analytically that a superposition of two counter-propagating waves with the same amplitude should simply result in $F=2 B(r, z) \cos (m \varphi) \cos (\omega t-G(r, z))$ i.e. a standing wave (since in this case the oscillatory term does not depend on $\varphi$, this situation represents a waveform characterized by maximum and minimum disturbances fixed in space with the minimum being continually replaced by the maximum and vice versa, as soon as $\cos (\omega t-G(r, z))$ changes its sign). In a similar way, mathematical developments for the case in which the amplitude of the two hydrothermal waves is not the same (i.e. a superposition with an arbitrary amplitude ratio $\eta$ $(0<\eta<1))$, led Kuhlmann and $\operatorname{Rath}^{50}$ to express the resulting flow as $F \cong B(r, z) \mathrm{a}(m \varphi) \cos [\mathrm{b}(m \varphi, G)-\omega t] \quad$ where $\quad \mathrm{a}(m \varphi)=\left[(1+\eta)^{2} \cos ^{2}\left(m \varphi-\varphi_{o}\right)\right.$ $\left.+(1-\eta)^{2} \sin ^{2}\left(m \varphi-\varphi_{o}\right)\right]^{1 / 2}$ and $\mathrm{b}(m \varphi, G)=\tan ^{-1}\left[\frac{1-\eta}{1+\eta} \tan \left(m \varphi-\varphi_{o}\right)\right]+G(r, z)$, which, because the oscillatory term depends on $\varphi$, should be regarded as an analytic representation of a wave travelling with the phase of the oscillations depending continuously on $\varphi$.

The most remarkable consequence of such theoretical developments is that, according to the first model (the standing wave resulting from the superposition of waves having the same amplitude), one should expect all the signals measured by thermocouples located at the same axial and radial coordinates (with different azimuthal positions) to be in phase or in phase opposition (only two 
values of phase shift being allowed, namely $\Delta G=0$ or $\Delta G=\pi$ ), whereas in the second case (different amplitude) the set of phase shifts of the resulting disturbance should not be discrete but continuous with $\Delta G$ depending linearly on the angular distance separating the thermocouples, provided such a distance $\Delta \varphi$ is shorter than $\pi / \mathrm{m}^{35}$.
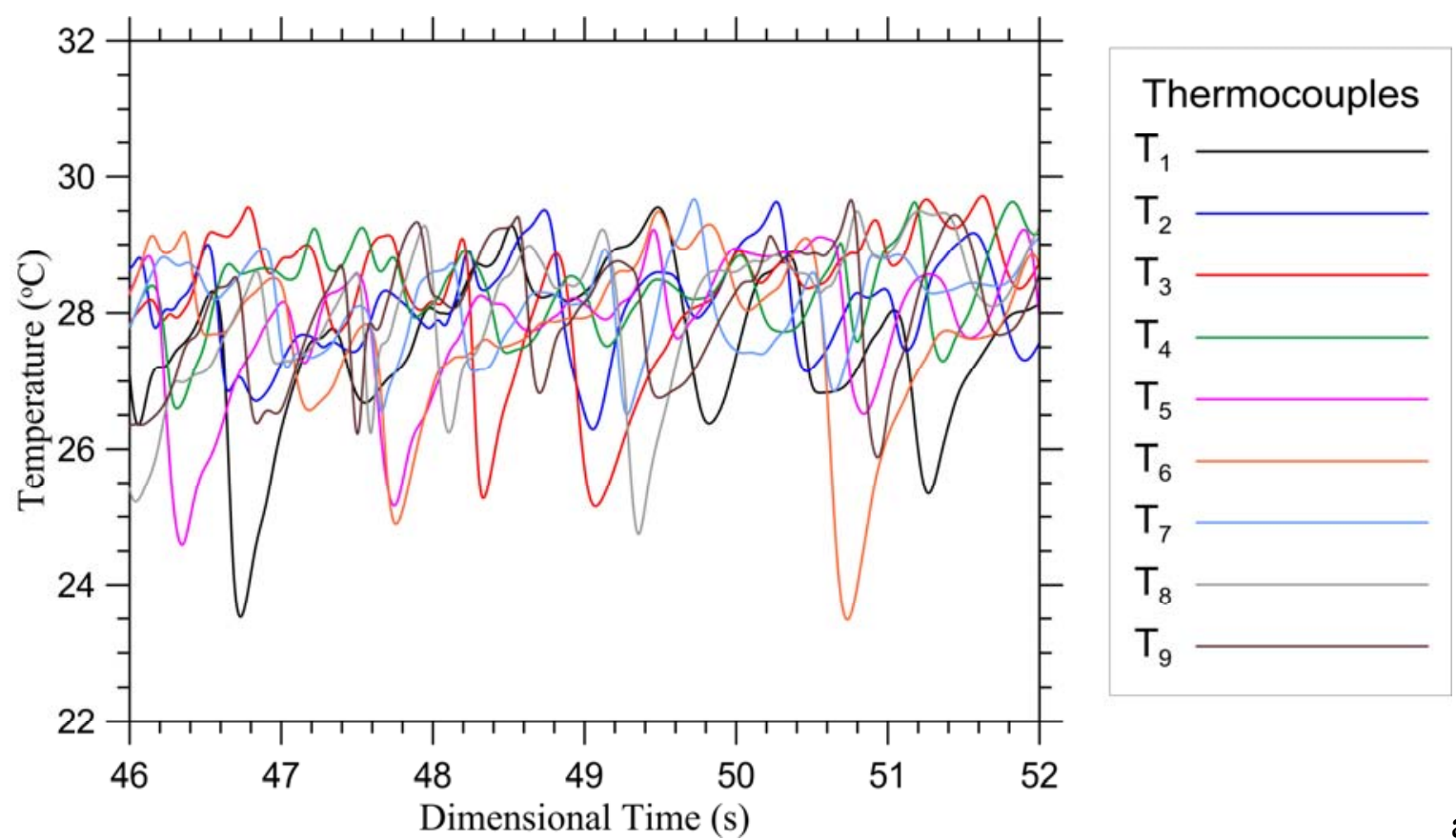

a)
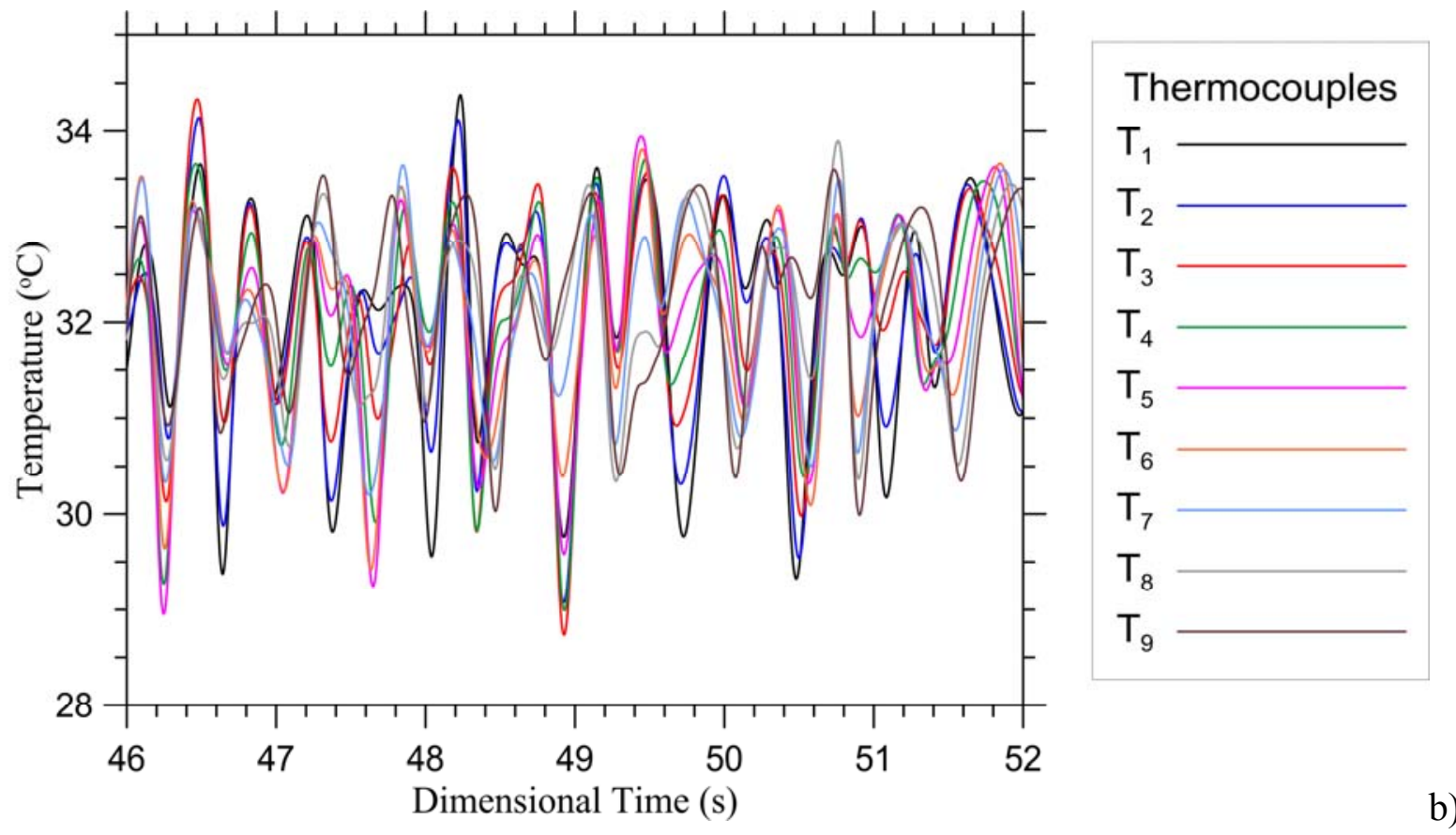

Figure 13: Signals provided by eight thermocouples at a fixed axial position evenly spaced along the azimuthal direction, as shown in Fig. $4 \mathrm{~b}\left(\mathrm{~A}_{\mathrm{F}}=0.63, \mathrm{Ma} \cong 2.3 \times 10^{4}, \mathrm{Ra} \cong 4.2 \times 10^{3}\right)$ : a) $\mathrm{z}=1 / 2$ (upper half), a continuous phase shift can be seen; b) $z=-1 / 2$ (lower half), only discrete values of phase shift are allowed. 
Although, rigorously speaking, the analytic relationship defined above applies to the simplified case of disturbances with a well-defined (fixed) value of the wavenumber (m), the related general principles can be easily extended to the case of a multi-wavenumber spectrum of disturbances. Indeed, the resulting signals (plotted versus time) should be yet expected to display recognizable clustering of maxima and minima for the pulsating case and vice versa significant scattering of such extrema in the rotating case [provided, the angular distance between subsequent thermocouples satisfies the criterion defined above, which for the present case involving values of the azimuthal wavenumber up to $\mathrm{m}=4$, would correspond to $\Delta \varphi<\pi / 4$, a requirement largely met by the nine evenly spaced probes shown in Fig. 4b].

The numerically computed signals provided by such thermocouples are shown in Fig. 13a and 13b for $z=1 / 2$ and $z=-1 / 2$, respectively. The strong amplitude variation experienced in time by these signals according to the azimuthal position of the probe clearly indicates that the amplitude of disturbances is not constant in time. The most interesting outcome of this figure, however, is the information it gives on the prevailing (pulsating or rotating) behavior of the flow.

Scattering of peaks for $z=1 / 2$ (Fig. 13a) as opposed to ordered accumulation of minima and maxima at certain temporal locations for $z=-1 / 2$ (Fig. 13b), illustrates that the different properties of the two facing toroidall rolls also have an impact on the selection of the dominant waveform. On the basis of such signals, indeed, it can be concluded that "on average" while the lower roll tends to support a pulsating behavior, the upper one promotes the superposition of waves resulting in an azimuthally propagating disturbance.
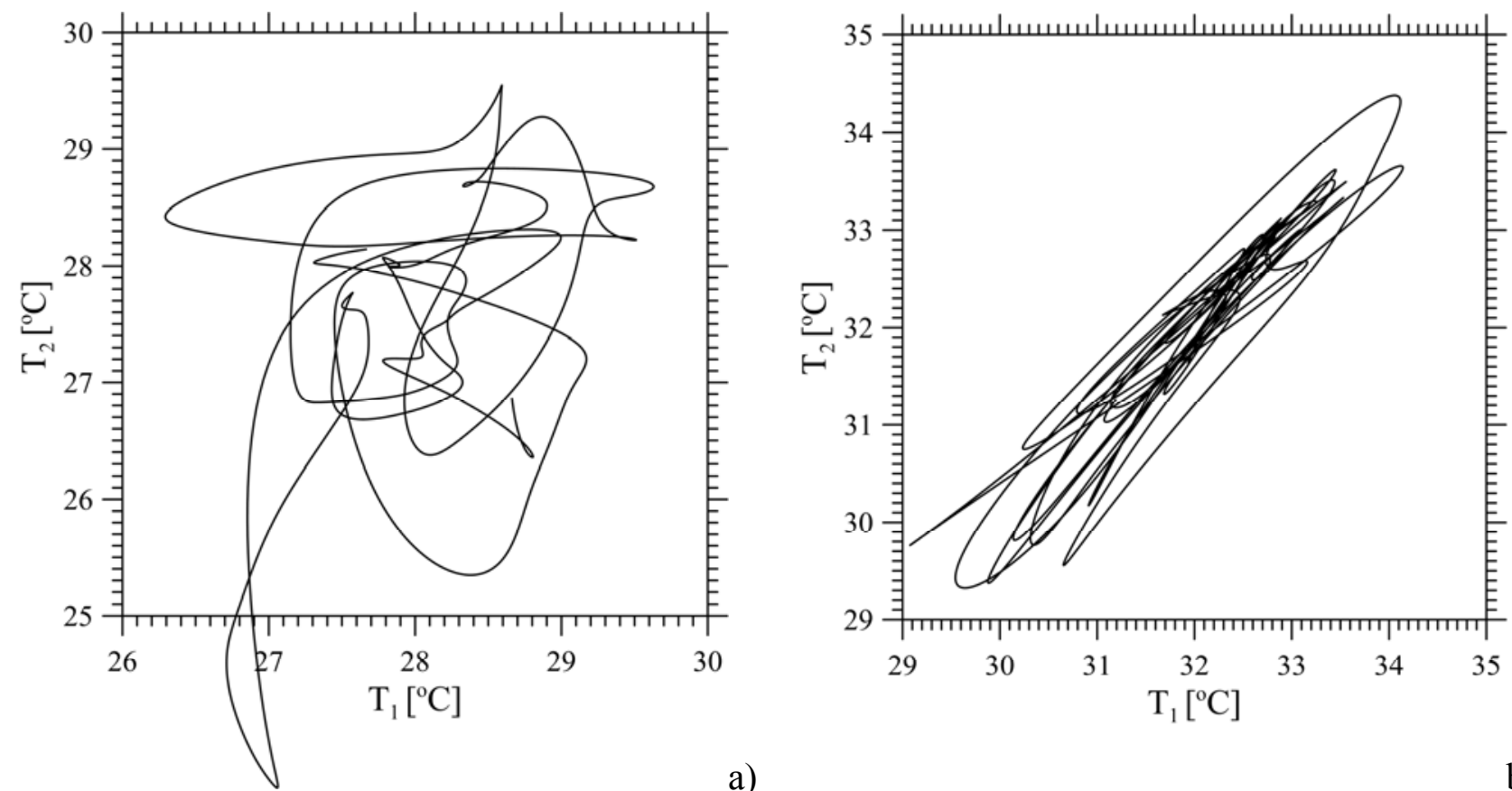

a)

Figure 14: Oscillatory behaviors in the phase space $\left(A_{F}=0.63, \mathrm{Ma} \cong 2.3 \times 10^{4}, \mathrm{Ra} \cong 4.2 \times 10^{3}\right)$ : a) $\mathrm{z}=1 / 2$ (upper half), b) $\mathrm{z}=-1 / 2$ (lower half). 
This feature can be made even more evident by taking a look at Fig. 14, where the oscillatory behavior as monitored by two close thermocouples satisfying the condition $\Delta \varphi<\pi / \mathrm{m}_{\max }$ has been represented in the phase space. This figure provides some additional meaningful information as it indicates that the behaviors elucidated above in terms of phase shift and amplitude of the signals correspond to well-defined "attractors" in the $\mathrm{T}_{\mathrm{i}}-\mathrm{T}_{\mathrm{j}}$ plane. Similar plots have been frequently used in the literature as idealized versions of the state of the considered system and its possible dynamic evolution.

For instance, looking at the interplay between $T_{2}$ and $T_{1}$, it becomes evident that in the first case (upper half), although the curve produced by plotting $\mathrm{T}_{2}$ as a function of $\mathrm{T}_{1}$ over the time has quite an irregular shape owing to the presence of many competing oscillatory modes (Fig. 15a), it circumscribes a region of finite (non-negligible) area. By contrast, in the second case (lower half), despite the complex spectrum of involved spatial modes and related frequencies (Fig. 15b), the resulting curve is much more regular, producing in the $\left(T_{1}, T_{2}\right)$ plane a sort of narrow band inclined with respect to the horizontal and vertical axes.
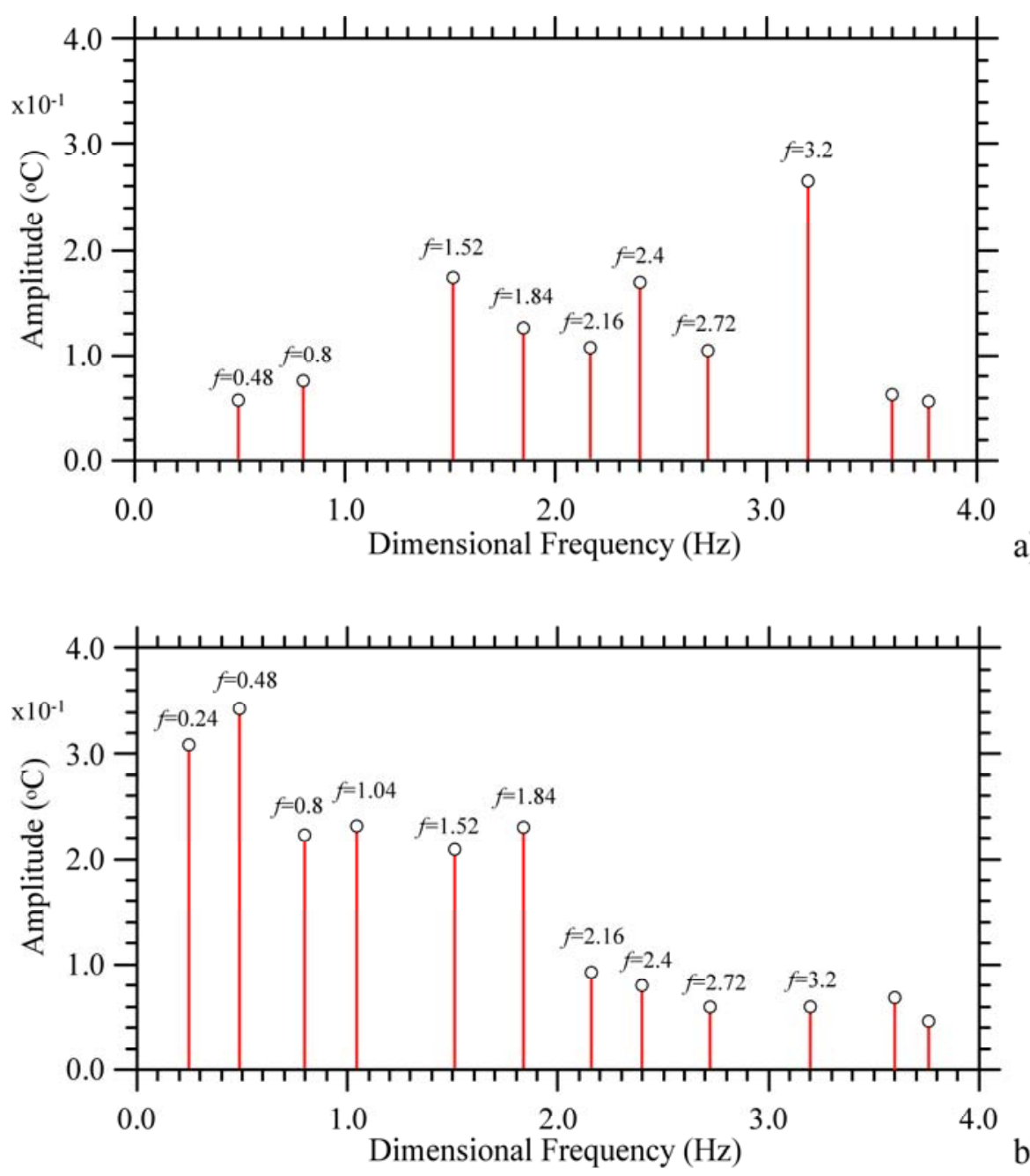

Figure 15: Typical frequency spectra of temperature signals: a) $z=1 / 2$ (upper half), b) $z=-1 / 2$ (lower half). 
Additional interesting insights can be obtained by examining the typical frequency spectrum of temperature signals in the upper and lower rolls (reported in Fig. 15a and 15b, respectively). Indeed, some notable differences can be clearly distinguished. While oscillatory modes with relatively high amplitudes are located in the medium and high-frequency part of the spectrum for $\mathrm{z}=-1 / 2$, disturbances cluster essentially in the low-frequency part for $z=1 / 2$. Moreover, on average, the amplitude of such modes versus the frequency displays a decreasing trend for the upper roll and, vice versa, an increasing behavior when the lower rolls is considered.

The existence of values of frequency common to both spectra, however, witnesses the expected interdependence or connection between rolls (as an example, the $f \cong 3.2 \mathrm{~Hz}$ frequency dominant in the lower roll is still present, though with a reduced amplitude, in the spectrum pertaining to $z=1 / 2$ where most of the high-amplitude modes are of a rotating kind; similarly some of the frequencies which are typical of the upper roll temperature signals, e.g., $f \cong 0.48 \mathrm{~Hz}$, survive in the frequency spectrum of the lower roll, where the set of high-amplitude disturbances comprises essentially pulsating modes).

Remarkably, the intriguing finding about the dominance of rotational or pulsating modes in the upper or lower toroidal roll, respectively, could have been anticipated (at least from a qualitative point of view) on the basis of earlier findings for the liquid bridge problem. As an example, in Lappa et al., ${ }^{35}$ standing waves were found to be long lasting for relatively shallow liquid bridges (where the toroidal roll takes a shape and morphology similar to that of the roll seen here in the lower half of the full zone), whereas they were observed to be quickly taken over by travelling waves for larger aspect ratios (rolls more extended in the axial direction).

Obviously, the present situation is much more complex because of the non-linear interaction among different disturbances with distinct azimuthal wavenumber that allows their relative amplitudes to change in time; moreover, the reciprocal influence of the two overlying and interpenetrating rolls evidenced by the frequency spectrum analysis, must be adequately taken into account. Perhaps the most relevant way to model these two influential factors would be to put a dependence on time directly in the parameter accounting for the relative amplitude of each couple of counterpropagating waves $\left(\eta_{m}\right)$ and to assume for such a ratio a proper dependence on the axial coordinate (perhaps, this observation might stimulate other researchers in this field to elaborate in the future a courageous and exhaustive analytical model along these lines).

\section{Conclusions}

So far, research activity dealing with the typical instabilities of Marangoni flow occurring during the processing of materials by the FZ method have been overwhelmingly devoted to the half-zone model (the so-called liquid bridge). Recently experiments by Kudo et al., ${ }^{33}$ revealed that an apparently chaotic state can emerge when the experiments are based on the full-zone configuration in a region of the space of parameters where on the basis of existing theories and earlier results for the classical liquid-bridge problem with high-Pr fluids, the flow should be relatively regular in time 
and with a simple structure in space (i.e. not too large supercritical conditions). Motivated by such unexpected experimental findings, a mathematical and numerical framework has been specifically elaborated to elucidate the spatial structure of fluid-dynamic disturbances, their nature and temporal behavior.

The present paper may be regarded as a natural continuation of an already existing full-zone-based line of inquiry where, however, the main focus was on liquid metals or semiconductor melts.

Starting from a cardinal concept, i.e. the possibility to model (or "view") the flow as two distinct overlying circulation systems, we have shown how the key to interpret the supercritical flow state lies essentially in the mental divisibility of the flow into two toroidal rolls with different intensity and morphological properties (due to the presence of buoyancy breaking the original system symmetry with respect to the equatorial plane).

A more in depth analysis of such dynamics has been based on a complementary (and less heuristic) approach based on the examination of the disturbances propagating along the circumferential direction, their superposition and the resulting behavior in terms of signals provided by probes located at specific positions in the fluid. A rich spectrum of convective dynamics has been revealed accordingly, consisting of modes with different spatial structure (wavenumber) appearing in isolated (single mode) or combined (hybrid state) form.

A possible categorization of the observed phenomena has been attempted according to the prevailing effect: the upper toroidal roll can be said to support a combination of travelling waves counter-propagating with different azimuthal wavenumbers and different amplitudes, whereas for the lower roll, the superposition of such waves results essentially in an oscillatory mechanism of a pulsating kind due to the similar amplitude with which waves of a given azimuthal wavenumber overlap. Moreover, for such a roll the spectrum of disturbances displays a much simpler structure, with a significant shrinkage of the number of modes involved.

The apparently "intermittent" response of the system is the consequence of the interference (nonlinear interaction) among all such modes. The related interconnected feedback loops and "iterative" processes make the system extremely sensitive to the interaction between the upper and lower rolls causing frequent switching from rotating to pulsating behaviors and intermediate (hybrid) situations in which the resulting scenario is apparently erratic.

Among the other questions being examined by the present paper we also included an attempt to address other points of critical importance to contemporary research in these fields, namely: 1) which percentage of what we have learned in the framework of earlier studies dealing with simplified configurations such as the liquid bridge can be applied or transferred to the proper interpretation of fluid-dynamic phenomena occurring in more complex and realistic configurations; and 2) what is the best (or most effective) practical and theoretical approach to investigate such apparently chaotic Marangoni flows or to help investigators to discern the complex interrelations among various aspects that are not independent of one another. 


\section{Acknowledgement}

We would like to thank Dr. Annalisa Riccardi for the kind support provided in executing the frequency spectrum analysis shown in Figure 15.

\section{References}

[1] Frank S., Schwabe D., (1997), Temporal and spatial elements of thermocapillary convection in floating zones, Exp. Fluids, 23: 234-251.

[2] Shevtsova V.M., Melnikov D.E., Legros J.C., (2001), Three-dimensional simulations of hydrodynamical instability in liquid bridges: influence of temperature-dependent viscosity, Phys Fluids, 13: 2851-2865.

[3] Shevtsova V.M., Melnikov D.E. and Legros J.C., (2003), Multistability of oscillatory thermocapillary convection in a liquid bridge, Phys. Rev. E, 68 (6): 066311 (14 pages).

[4] Shevtsova V., Melnikov D.E., Nepomnyashchy A., (2009), New flow regimes generated by mode coupling in buoyant-thermocapillary convection, Phys. Rev. Lett., 102: 134503.

[5] Shevtsova V., Mialdun A., Kawamura H., Ueno I., Nishino K. and Lappa M., (2011), Onset of Hydrothermal Instability in Liquid Bridge. Experimental Benchmark, Fluid Dyn. Mater. Process, 7(1): $1-28$

[6] Melnikov D.E., Shevtsova V.M. and Legros J.C., (2005), Route to aperiodicity followed by high Prandtl-number liquid bridge. 1-g case, Acta Astronautica, 56(6): 601-611.

[7] Benz K.W., (1990), Factors controlling crystal perfections during growth under microgravity, Proceedings VIIth European Symposium on Materials and Fluid Sciences in Microgravity Oxford (United Kingdom), 10/15 September, ESA SP 295: 59-61.

[8] Cröll A., Kaiser Th., Schweizer M., Danilewsky A.N., Lauer S., Tegetmeier A., Benz K.W., (1998), Floating-zone and floating-solution-zone growth of GaSb under microgravity, J. Cryst. Growth, 191: 365-376.

[9] Saurat M. and Revcolevschi A., (1971), Preparation by the floating zone method of refractory oxide monocrystals, in particular gallium oxide, and study of some of their properties, Revue Internationale des Haute Temperatures et des Refractaires 8, 291-304.

[10] Shindo I., Ii N., Kitamura K., Kimura S., (1979), Single crystal growth of substituted yttrium iron garnets $\mathrm{Y}_{3} \mathrm{Fe}_{5}-x(\mathrm{Ga}, \mathrm{Al})_{x} \mathrm{O}_{12}$ by the floating zone method, J. Cryst. Growth, 46, 307-313.

[11] Shindo I., (1980), Determination of the phase diagram by the slow cooling float zone method: The system MgO-TiO 2 , J. Cryst. Growth, 50, 839-851.

[12] Balbashov A.M. and Egorov S.K., (1981), Apparatus for growth of single crystals of oxide compounds by floating zone melting with radiation heating, J. Cryst. Growth, 52, 498-504.

[13] Kimura S. and Kitamura K., (1992),Floating zone crystal growth and phase equilibria: A review, J. Am. Ceram. Soc., 75(6), 1140-1146.

[14] Revcolevschi A. and Jegoudez J., (1997), Growth of large high-Tc single crystals by the floating zone method: A review, Progr. Mater. Sci. 42, 321-339.

[15] Moest B., Glebovsky V.G., Brongersma H.H., Bergmans R.H., Denier van der Gon A.W., Semenov V.N., (1998), Study of Pd single crystals grown by crucibleless zone melting, J. Cryst. Growth, 192(3-4), 410-416.

[16] Villora E.G., Shimamura K., Yoshikawa Y., Aoki K., Ichinose N., (2004), Large-size $\beta-\mathrm{Ga}_{2} \mathrm{O}_{3}$ single crystals and wafers, J. Cryst. Growth, 270, 420-426.

[17] Balbashov A.M., Tsvetkova A.A., Chervonenkis A.Y., (1975), Imperfections in crystals of yttrium-iron garnet grown from nonstoichiometric melts, Neorg. Mater., 11, 108-111.

[18] Higuchi M. and Kodaira K., (1992), Effect of $\mathrm{ZrO}_{2}$ addition on FZ growth of rutile single crystals, J. Cryst. Growth, 123, 495-499. 
[19] Dabkowska H. A. and Dabkowski A. B., (2010), Crystal Growth of Oxides by Optical Floating Zone Technique, in Springer Handbook of Crystal Growth 367 (2010), pp. 367-391

[20] Dabkowska H.A. and Gaulin B. D., Crystal Growth of Technologically Important Electronic Materials, ed. by K. Byrappa, T. Ochachi, M. Klapper, R. Fornari (Allied Publishers PVT, New Delhi 2003) pp. 341-354

[21] Baumgartl J., Gewald M., Rupp R., Stierlen J., Muller G., (1990), The use of magnetic fields and microgravity in melt growth of semiconductors : a comparative study, Proceedings VIIth European Symposium on Materials and Fluid Sciences in Microgravity Oxford (United Kingdom), 10/15 September, ESA SP 295.

[22] Lappa M., (2003), Three-dimensional numerical simulation of Marangoni flow instabilities in floating zones laterally heated by an equatorial ring, Phys. Fluids, 15(3): 776-789.

[23] Lappa M., (2004), Combined effect of volume and gravity on the three-dimensional flow instability in non-cylindrical floating zones heated by an equatorial ring, Phys. Fluids, 16(2): 331343.

[24] Lappa M., (2004), Floating zones heated around the equatorial plane: models and simulations, Microgravity Sci. Tech., XV/3: 36-51.

[25] Lappa M., (2005), Analysis of flow instabilities in convex and concave floating zones heated by an equatorial ring under microgravity conditions, Computers \& Fluids, 34(6): 743-770.

[26] Minakuchi, H., Okano, Y. and Dost, S., (2004), A three-dimensional numerical simulation study of the Marangoni convection occurring in the crystal growth of $\mathrm{Si}_{\mathrm{x}} \mathrm{Ge}_{1-\mathrm{x}}$ by the floating zone technique in zero gravity, J. Cryst. Growth, 266: 140-144.

[27] Houchens B. C. and Walker J. S., (2005), Modeling the floating zone: Instabilities in the half zone and full zone, Journal of Thermophysics and Heat Transfer, 19(2): 186-198.

[28] Gelfgat A.Yu., Rubinov A., Bar-Yoseph P.Z. and Solan A., (2005), On the Three-Dimensional Instability of Thermocapillary Convection in Arbitrarily Heated Floating Zones in Microgravity Environment, Fluid Dyn. Mater. Process, 1(1): 21-32.

[29] Lin K., Dold P. and. Benz K.W, (2007), Numerical simulation of general flow features in a germanium floating zone, Computational Materials Science, 39(2): 424-429

[30] Chen C.J. and Chieh H., (1995), Measurement of the float-zone interface shape for lithium niobate, J. Cryst. Growth, 149, 87-95.

[31] Lan C.W., (2003), Three-dimensional simulation of floating-zone crystal growth of oxide crystals, J. Cryst. Growth, 247, 597-612.

[32] Bouizi O., Delcarte C., Kasperski G., (2007), Stability study of the floating zone with respect to the Prandtl number value, Phys. Fluids 19, 114102

[33] Kudo M., Ueno I. and Kawamura H., (2014), Transition of thermocapillary convection in a full-zone liquid bridge, Transactions of the JSME (in Japanese), 80(812) (15 pages), [DOI: 10.1299/transjsme.2014tep0095]

[34] Sakurai M., Tamura A., Kinoshita A. and Hirata A., (1998), Marangoni convection in a liquid bridge that is heated by a ring heater in a full zone model, Journal of The Japan Society of Microgravity Application, 15: 419-424.

[35] Lappa M., Savino R. and Monti R., (2001), Three-dimensional numerical simulation of Marangoni instabilities in liquid bridges: influence of geometrical aspect ratio, Int. J. Num. Meth. Fluids, 36(1): 53-90.

[36] Otani S., Tanaka T., Ishizawa Y., (1988), Control of heat flow to feed rod in floating zone system, J. Cryst. Growth, 87, 175-179.

[37] Rivas D. and Vazquez-Espi C., (2001), An analysis of lamp irradiation in ellipsoidal mirror furnaces, J. Cryst. Growth, 223, 433-445.

[38] Harlow F.H. and Welch J.E. (1965), Numerical calculation of time-dependent viscous incompressible flow with free surface, Phys. Fluids, 8: 2182-2189.

[39] Chorin A.J., (1968), Numerical solutions of the Navier-Stokes equations, Math. Comput., 22: 745-762. 
[40] Temam R., (1968), Une méthode d'approximation de la solution des équations de NavierStokes, Bull. Soc. Math. France, 98: 115-152.

[41] Ladyzhenskaya O.A., (1969), The Mathematical Theory of Viscous Incompressible Flow, Gordon and Breach, $2^{\text {nd }}$ Edition, New York - London, 1969.

[42] Lappa M. and Savino R., (1999), Parallel solution of the three-dimensional Marangoni flow instabilities in liquid bridges, Int. J. Num. Meth. Fluids, 31(6): 911-925.

[43] Melnikov D., Pushkin D., and Shevtsova V., (2011), Accumulation of particles in timedependent thermocapillary flow in a liquid bridge. Modeling of experiments, Eur. Phys. J. Special Topics, 192, 29-39.

[44] Lappa M., (2013), On the variety of particle accumulation structures under the effect of gjitters, J. Fluid Mech., 726: 160-195.

[45] Assenheimer M. and Steinberg V., (1996), Observation of Coexisting Upflow and Downflow Hexagons in Boussinesq Rayleigh-Bénard Convection, Phys. Rev. Lett., 76: 756-759.

[46] Cakmur R.V., Egolf D.A., Plapp B.B. and Bodenschatz E., (1997), Bistability and competition of spatiotemporal chaotic and fixed point attractors in Rayleigh-Bénard convection, Phys. Rev. Lett., 79(10): 1853-1856.

[47] Johnson D. and Narayanan R., (1996), Experimental observation of dynamic mode switching in interfacial-tension-driven convection near a codimension-two point, Phys. Rev. E 54, R3102(R).

[48] Dauby P. C., Lebon G., and Bouhy E., (1997), Linear Bénard-Marangoni instability in rigid circular containers, Phys. Rev. E 56, 520

[49] Gelfgat A.Yu., Bar-Yoseph P.Z., Solan A., (2000), Axisymmetry breaking instabilities of Natural convection in a vertical Bridgman growth configuration, J. Cryst. Growth, 220: 316-325.

[50] Kuhlmann H.C. and Rath H.J., (1993), On the interpretation of phase measurements of oscillatory thermocapillary convection in liquid bridges, Phys. Fluids A 5 (9): 2117-2120. 